TRANSACTIONS OF THE

AMERICAN MATHEMATICAL SOCIETY

Volume 351, Number 4, April 1999, Pages 1507-1530

S 0002-9947(99)02113-3

\title{
LIMIT SETS OF DISCRETE GROUPS OF ISOMETRIES OF EXOTIC HYPERBOLIC SPACES
}

\author{
KEVIN CORLETTE AND ALESSANDRA IOZZI
}

\begin{abstract}
Let $\Gamma$ be a geometrically finite discrete group of isometries of hyperbolic space $\mathcal{H}_{\mathbb{F}}^{n}$, where $\mathbb{F}=\mathbb{R}, \mathbb{C}, \mathbb{H}$ or $\mathbb{O}$ (in which case $n=2$ ). We prove that the critical exponent of $\Gamma$ equals the Hausdorff dimension of the limit sets $\Lambda(\Gamma)$ and that the smallest eigenvalue of the Laplacian acting on square integrable functions is a quadratic function of either of them (when they are sufficiently large). A generalization of Hopf ergodicity theorem for the geodesic flow with respect to the Bowen-Margulis measure is also proven.
\end{abstract}

\section{INTRODUCTION}

In a previous paper $[\mathrm{C}]$, the first author studied limit sets of discrete groups of isometries of rank one symmetric spaces of noncompact type. These symmetric spaces are the hyperbolic spaces associated with the reals, complexes, quaternions, or Cayley numbers. The main point of interest in that paper was the Hausdorff dimension (in an appropriate sense) of the limit set and its relationships with other invariants, such as the smallest eigenvalue of the Laplacian acting on squareintegrable functions and the exponent of growth of the group. In the cases where the group of all isometries of the symmetric space has Kazhdan's property, this led to conclusions about limitations on the possible exponents of growth, among other things. The most precise results were obtained for a class of groups which, in that paper, were called geometrically cocompact; these groups are also known as convex cocompact groups, or geometrically finite groups without cusps. In particular, the exponent of growth was shown to be equal to the Hausdorff dimension of the limit set, from which one could draw conclusions about the topological structure of the hyperbolic manifolds corresponding to such groups.

In this paper, we will study the case of arbitrary geometrically finite groups of isometries of these symmetric spaces. The main result is that the exponent of growth coincides with the Hausdorff dimension of the limit set; as a consequence, when these are sufficiently large, the smallest eigenvalue of the Laplacian is a quadratic function of either of them and can be shown to correspond to a square-integrable eigenfunction. (This latter fact will be exploited in a forthcoming paper of the first author to give estimates on the topological codimension of the

Received by the editors February 27, 1995 and, in revised form, April 15, 1997.

1991 Mathematics Subject Classification. Primary 58F11; Secondary 53C35, 58F17.

K. C. received support from a Sloan Foundation Fellowship, an NSF Presidential Young Investigator award, and NSF grant DMS-9203765. A. I. received support from NSF grants DMS 9001959, DMS 9100383 and DMS 8505550.

(C)1999 American Mathematical Society 
limit sets of geometrically finite groups in the quaternionic and Cayley cases which refine Corollary 6.4 of this paper.) We will also prove a generalization of the Hopf ergodicity theorem for the geodesic flow.

We should remark that the equality between the critical exponent of the Poincaré series and the Hausdorff dimension of the limit set has been proven independently in $[\mathrm{Bi}-\mathrm{J}]$ in the case of a general non-elementary finitely generated Kleinian group (or more generally for any discrete group of Möbius transformations acting on the hyperbolic ball in any dimension in the general rank 1 case). Also, partial results on ergodicity of the geodesic flow and the correspondence among the above invariants have been obtained in $[\mathrm{Y}]$ in the more general case of a Riemannian manifold with non-constant pinched negative curvature. Our techniques however exploit the structure of the hyperbolic space, since we use here a family of conformally equivalent sub-Riemannian metrics at infinity. As a consequence of this different approach, we can complete the picture of the above result in our case; the general case is still unknown and, as far as we can say, quite inaccessible.

Of course, these questions have already been studied for geometrically finite Fuchsian groups by Patterson $[\mathrm{Pt}]$ and for geometrically finite Kleinian groups by Sullivan [Su1], [Su2]. Our approach is based in outline on that of Sullivan, but certain parts of the argument require considerably more attention due to the more complicated geometry of the spaces under consideration. The argument differs from the case of geometrically cocompact groups, where one can show that the PattersonSullivan measure coincides with Hausdorff measure. The question of whether or not the Patterson-Sullivan measure coincides with Hausdorff measure in the case of geometrically finite groups is more delicate. Sullivan has shown that there are cases in which they do not coincide; to remedy this, he was led to consider measures defined by packing rather than covering constructions. We have not attempted to elucidate these issues in this paper.

Since one cannot rely on the equivalence of the Patterson-Sullivan measure and Hausdorff measure, it is necessary to follow a more circuitous route to establish our results. In $\S 2$, we will show that the limit set of a geometrically finite group is the union of the radial limit set and a countable number of parabolic fixed points. This, combined with the results of $[\mathrm{C}]$, already shows that the critical exponent gives an upper bound for the Hausdorff dimension of the limit set. In $\S 3$, we show that the Patterson-Sullivan measure has no atoms in the case of a geometrically finite group. In the course of this argument, we give a corrected version of a result stated in $[\mathrm{Au}]$. In $\S 4$, we use the Patterson-Sullivan measure to construct an invariant measure for the geodesic flow. We show that this measure is finite and (in §5) ergodic. Finally, in $\S 6$, we arrive at the conclusion that the Hausdorff dimension of the limit set is equal to the critical exponent and deduce that either of these is a quadratic function of the smallest eigenvalue of the Laplacian. Consequently, one finds by the same method as in $[\mathrm{C}]$ that a geometrically finite quaternionic or Cayley hyperbolic manifold has at most one end which is not a cusp.

The second author thanks Jonathan Poritz for useful conversations. We both thank the Institute for Advanced Study, the Mathematical Sciences Research Institute and the Mathematics Research Centre at the University of Warwick, where parts of this work were done. We would especially like to express our appreciation to Gabriele for inspirational remarks during some of the dark moments which haunted this work. 


\section{The Limit SET}

Let $\mathcal{H}_{\mathbb{F}}^{n}$ be the $n$-dimensional hyperbolic space, where $\mathbb{F}=\mathbb{R}$ the reals, $\mathbb{F}=\mathbb{C}$ the complex numbers, $\mathbb{F}=\mathbb{H}$ the quaternions or $\mathbb{F}=\mathbb{O}$ the octonians (in which case $n=2$ ). It is well known that in all of these cases $\mathcal{H}_{\mathbb{F}}^{n}$ is a simply connected Riemannian manifold with sectional curvature pinched between -4 and -1 and can be identified as a homogeneous space with $G / K$, where $G=O_{\mathbb{F}}(1, n)$ is the full group of isometries and $K$ is a maximal compact subgroup. (In the following we shall often refer to the Iwasawa decomposition $K A N$ of $G$ and to the Langlands decomposition $M A N$ of a parabolic subgroup of $G$.) The sphere at infinity of $\mathcal{H}_{\mathbb{F}}^{n}$, denoted by $\partial \mathcal{H}_{\mathbb{F}}^{n}$, can be identified with a $(k n-1)$-dimensional sphere, where $k=\operatorname{dim}_{\mathbb{R}}(\mathbb{F})$, and we write $\overline{\mathcal{H}_{\mathbb{F}}^{n}}=\mathcal{H}_{\mathbb{F}}^{n} \cup \partial \mathcal{H}_{\mathbb{F}}^{n}$. If $\Gamma \subset O_{\mathbb{F}}(1, n)$ is a discrete subgroup and $x \in \mathcal{H}_{\mathbb{F}}^{n}$, let $L_{\Gamma}=\overline{\Gamma \cdot x} \cap \partial \mathcal{H}_{\mathbb{F}}^{n}$ denote the limit set of $\Gamma$ (which can be proven to be independent of $x$, see [E-O]). The radial limit set $L_{\Gamma}^{\mathrm{rad}}$ of $\Gamma$ is a subset of $L_{\Gamma}$ consisting of all points $\xi \in L_{\Gamma}$ such that the orbit $\Gamma \cdot x$ intersects some $r$ neighborhood of any geodesic ray approaching $\xi$ in an infinite number of points. (Note that this definition of radial limit set is weaker than the one used in $[\mathrm{C}]$. However, all the results we are going to use from $[\mathrm{C}]$ hold true even with this weaker definition.) Let $O_{\Gamma}=\partial \mathcal{H}_{\mathbb{F}}^{n} \backslash L_{\Gamma}$ be the ordinary set of $\Gamma$ (sometimes referred to as the domain of discontinuity); the action of $\Gamma$ on $\mathcal{H}_{\mathbb{F}}^{n} \cup O_{\Gamma}$ is properly discontinuous, [E-O], and we denote by $\bar{M}_{\Gamma}=\mathcal{H}_{\mathbb{F}}^{n} \cup O_{\Gamma} / \Gamma$ the associated Kleinian manifold. We shall also set $M_{\Gamma}=\mathcal{H}_{\mathbb{F}}^{n} / \Gamma$.

Definition 2.1. Let $\operatorname{Hull}\left(L_{\Gamma}\right)$ be the convex hull of the limit set $L_{\Gamma}$. $\Gamma$ is geometrically finite if it is finitely generated and if, for every $\epsilon>0$, the volume of an $\epsilon$-neighborhood of $\operatorname{Hull}\left(L_{\Gamma}\right) / \Gamma$ is finite $([\mathrm{Ad}],[\mathrm{Bw}])$.

In the following we shall also assume that $\Gamma$ is torsion-free (passing, if necessary, to a subgroup of finite index) and that $\Gamma$ is not elementary, that is there are no $\Gamma$-fixed points in $\mathcal{H}_{\mathbb{F}}^{n} \cup \partial \mathcal{H}_{\mathbb{F}}^{n}$. Because of the absence of torsion, this amounts to assuming that $\Gamma$ is not contained in a parabolic subgroup of $O_{\mathbb{F}}(1, n)$. (Note: the notion of parabolic subgroup used here is that of the theory of algebraic groups; this should not be confused with the notion of a discrete parabolic group, defined in 2.2 below.)

If $K$ is a maximal compact subgroup of $O_{\mathbb{F}}(1, n)$, it is possible to describe a family of conformally equivalent $K$-invariant sub-Riemannian metrics on $\partial \mathcal{H}_{\mathbb{F}}^{n}$, which are the restrictions of $K$-invariant Riemannian metrics on $\partial \mathcal{H}_{\mathbb{F}}^{n}$ to the subbundle of the tangent bundle defined as the orthogonal complement of the vertical tangent bundle of the Hopf fibration $\partial \mathcal{H}_{\mathbb{F}}^{n} \rightarrow \mathbb{P}_{\mathbb{F}}^{n-1}$ (see [C], [Gr], [Mi], [Mo], [Pn1], [Pn2], [Pn3], [St] for details). Then, given any set $C \subset \partial \mathcal{H}_{\mathbb{F}}^{n}$, we can define the $\delta$-dimensional Hausdorff measure of $C$ by

$$
H^{\delta}(C)=\lim _{R \rightarrow 0} \inf _{\mathcal{U}} \sum_{B(\xi, r) \in \mathcal{U}} r^{\delta}
$$

where $\mathcal{U}$ ranges over all countable coverings of $C$ by balls $B(\xi, r)$ centered at $\xi$ of radius $r<R$. Then the Hausdorff dimension of $C$ is $H-\operatorname{dim}(C)=\inf \left\{\delta: H^{\delta}(C)=\right.$ $0\}$.

It is well known (see [C, Proposition 3.2] for example) that for geometrically cocompact discrete groups of isometries (that is groups for which $\bar{M}_{\Gamma}$ is compact) the radial limit set coincides with the full limit set. For geometrically finite groups 
this is not true any more, but we have a corresponding result. Let us start with the following definition.

Definition 2.2. Let $\Gamma_{1}$ be an infinite subgroup of a group of isometries $\Gamma$ of $\mathcal{H}_{\mathbb{F}}^{n}$. Then

1. $\Gamma_{1}$ is parabolic if the set of fixed points of $\Gamma_{1}$ consists of a single point $\xi \in \partial \mathcal{H}_{\mathbb{F}}^{n}$ and $\Gamma_{1}$ preserves setwise any horosphere based at $\xi$. Any point $\xi$ arising in this way as the unique fixed point of a subgroup $\Gamma_{1} \subset \Gamma$ is called a parabolic fixed point of $\Gamma$;

2. $\Gamma_{1}$ is loxodromic if it contains a loxodromic element, that is an element whose set of fixed points consists of two distinct elements $\xi, \eta \in \partial \mathcal{H}_{\mathbb{F}}^{n}$, and $\Gamma_{1}$ preserves setwise the geodesic connecting $\xi$ and $\eta$.

Then we have

Theorem 2.3. If $\Gamma$ is geometrically finite, the limit set consists of the radial limit set together with a countable number of parabolic fixed points.

Corollary 2.4. If $\Gamma$ if geometrically finite, then $H-\operatorname{dim}\left(L_{\Gamma}\right)=H-\operatorname{dim}\left(L_{\Gamma}^{\text {rad }}\right)$.

Proof of Theorem 2.3. It is immediate from the two following facts and the definition of Hausdorff dimension.

Lemma 2.5. There are finitely many $\Gamma$-orbits of parabolic fixed points.

Lemma 2.6. Any non-parabolic limit point is radial.

The proof of both of these facts follows from Margulis' lemma and the "thickthin" decomposition of $M_{\Gamma}$. We briefly recall these here and we refer to [Bw] for further details.

Proposition 2.7 (Margulis' Lemma). For any integer $n$ and for $\kappa \geq 1$, there exists a constant $\epsilon(n, \kappa)>0$ such that if $X$ is an $n$-dimensional manifold with sectional curvature between $-\kappa^{2}$ and $-1, \Gamma \subset I s o(X)$ is discrete and $x \in X$, then the group $\Gamma_{\epsilon}(x)$ generated by $\{\gamma \in \Gamma: d(x, \gamma x) \leq \epsilon\}$ is almost nilpotent for $\epsilon \leq \epsilon(n, \kappa)$ (that is $\Gamma_{\epsilon}(x)$ contains a nilpotent subgroup of finite index). The constant $\epsilon(n, \kappa)$ is called the Margulis constant.

Let

$$
M_{\Gamma}^{\epsilon-t h i n}=\left\{p \in M_{\Gamma}: \text { injectivity radius at } p \leq \epsilon\right\}
$$

and

$$
M_{\Gamma}^{\epsilon-\text { thick }}=\left\{p \in M_{\Gamma}: \text { injectivity radius at } p \geq \epsilon\right\} .
$$

Then, for $\epsilon=\epsilon(n, \kappa), M_{\Gamma}^{\epsilon-t h i n}$ is the disjoint union of connected components, each of which has the form $T_{\epsilon}(\tilde{\Gamma}) / \tilde{\Gamma}$, where $\tilde{\Gamma}$ is a maximal infinite elementary subgroup of $\Gamma$ and $T_{\epsilon}(\tilde{\Gamma})=\left\{x \in \mathcal{H}_{\mathbb{F}}^{n}: \tilde{\Gamma}_{\epsilon}(x)\right.$ is infinite $\}$; the groups $\tilde{\Gamma}$ can be either parabolic or loxodromic and, choosing appropriately $\epsilon \leq \epsilon(n, \kappa)$, we can arrange things in such a way that all $\tilde{\Gamma}$ 's are parabolic. In this case the $T_{\epsilon}(\tilde{\Gamma})$ 's are horoballs based at points $\xi \in \partial \mathcal{H}_{\mathbb{F}}^{n}$, and the $\tilde{\Gamma}$ 's are the stabilizers of such parabolic points. A priori there could be an infinite number of such cusps; however, because $\Gamma$ is geometrically finite, the intersection of $M_{\Gamma}^{\epsilon-t h i c k}$ with the convex hull of the limit set has to be compact, and hence there are only finitely many components in $M_{\Gamma}^{\epsilon-t h i n}$, thus proving Lemma 2.5. 
Proof of Lemma 2.6. Let $\sigma$ be a geodesic ray contained in the convex hull of $L_{\Gamma}$ and heading towards $\xi \in L_{\Gamma}$. Let $\bar{\sigma}$ be its projection onto $M_{\Gamma}$. If $\bar{\sigma}$ reenters $M_{\Gamma}^{\epsilon-\text { thick }}$ infinitely often, then $\xi$ is radial. If not, it is eventually contained in a component of $M_{\Gamma}^{\epsilon-t h i n}$, so $\sigma$ is contained in a horoball centered at $\xi$, which implies that $\xi$ is parabolic.

\section{The Patterson-Sullivan measure}

We recall here the definitions of critical exponent of $\Gamma$ and of Patterson-Sullivan measure; for a more detailed account, see [C] (see also [Pt] and [Su1] for the real case).

Given any discrete subgroup $\Gamma \subset O_{\mathbb{F}}(1, n)$, its critical exponent $\delta_{\Gamma}$ is the infimum of all $s$ for which the Poincaré series

$$
P_{s}(x, y)=\sum_{\gamma \in \Gamma} e^{-s d(x, \gamma y)}
$$

converges for some $x, y \in \mathcal{H}_{\mathbb{F}}^{n}$. Define now a family of measures by

$$
\mu_{s, x}=\frac{\sum_{\gamma \in \Gamma} e^{-s d(x, \gamma y)} \theta_{\gamma y}}{\sum_{\gamma \in \Gamma} e^{-s d(O, \gamma y)}}
$$

where $\theta_{\gamma y}$ is the point mass measure at $\gamma y \in \mathcal{H}_{\mathbb{F}}^{n}$ and $O$ is a fixed reference point. Then the Patterson Sullivan measure is defined as the weak limit $\mu_{x}=\lim _{s \rightarrow \delta_{\Gamma}^{+}} \mu_{s, x}$ and is a finite measure supported on $L_{\Gamma}$ (if necessary after the choice of a sequence $\left\{s_{i}\right\} \rightarrow \delta_{\Gamma}^{+}$and some minor adjustments $[\mathrm{Pt}]$ to assure the divergence of the denominator in the definition of $\left.\mu_{x, s}\right)$. It is proven in [C] that the Patterson-Sullivan measure has the property that if $x, y \in \mathcal{H}_{\mathbb{F}}^{n}$, then $\mu_{x}=e^{\delta_{\Gamma}\left(b_{\xi}(y)-b_{\xi}(x)\right)} \mu_{y}$, where $b_{\xi}(x)$ is the Busemann function defined as

$$
b_{\xi}(x)=\lim _{t \rightarrow \infty}(d(x, \sigma(t))-t)
$$

where $\sigma:[0, \infty) \rightarrow \mathcal{H}_{\mathbb{F}}^{n}$ is a geodesic ray asymptotic to $\xi \in \partial \mathcal{H}_{\mathbb{F}}^{n}$. Moreover, if $\gamma \in \Gamma$, then $\gamma^{*} \mu_{x}=\mu_{\gamma x}$. Observe also that if $x, y \in \mathcal{H}_{\mathbb{F}}^{n}$, then

$$
b_{\xi}(x)-b_{\xi}(y)=\lim _{u \rightarrow \xi}(d(x, u)-d(y, u)) \leq d(x, y),
$$

where equality holds if $x, y, u$ all belong to the same geodesic headed towards $\xi$.

The main purpose of this section will be to show that the Patterson-Sullivan measure has no atoms. We start with the following lemma which will be used several times in the proof of Theorem 3.2 and elsewhere in the paper.

Lemma 3.1. For any $\epsilon>0$, there is some constant $C$ such that for any geodesic triangle in $\mathcal{H}_{\mathbb{F}}^{n}$ with sides $a, b, c$ and opposite angles $\alpha, \beta, \gamma$ with $\gamma \geq \epsilon$ we have $a+b-C \leq c \leq a+b$.

Proof. The second inequality is just the triangle inequality. Toponogov's theorem implies that $c$ is bounded below by the distance between the endpoints of two geodesic segments of lengths $a, b$ separated by an angle $\gamma$ in the real hyperbolic space with curvature -1 , so we reduce to this case. This now becomes a computation in hyperbolic trigonometry. Observe first that if $\cosh c=y$, then $c=$ $\ln \left(y+\sqrt{y^{2}-1}\right) \geq \ln y$. Hence, from the hyperbolic law of cosines

$$
\cosh c \geq \cosh a \cosh b-\sinh a \sinh b \cos \gamma,
$$


it follows that

$$
\begin{aligned}
c & \geq \ln (\cosh a \cosh b-\sinh a \sinh b \cos \gamma) \\
& \geq \ln ((1-|\cos \gamma|) \cosh a \cosh b) .
\end{aligned}
$$

Since $\cosh a \geq e^{a}$, then $c \geq \ln (1-|\cos \gamma|)+a+b$.

Theorem 3.2. $\mu_{x}$ has no atoms on the parabolic fixed points. In particular, $\mu_{x}\left(L_{\Gamma}\right)$ $=\mu_{x}\left(L_{\Gamma}^{r a d}\right)$.

Proof. The strategy of the proof will consist in finding estimates on the total mass of $\mu_{x}$ along geodesics approaching the cusps and then showing, under the assumption that some cusp in $M_{\Gamma}$ is an atom for $\mu_{x}$, that such estimates lead to a contradiction. Let $C$ be a cusp in $M_{\Gamma}$ and $\hat{C} \subset \mathcal{H}_{\mathbb{F}}^{n}$ be a corresponding disjoint union of horoballs. Let us consider the half-space model for $\mathcal{H}_{\mathbb{F}}^{n}$ in such a way that the base point of a horoball $B \subset \hat{C}$ corresponds to the point at infinity. To simplify the exposition we will break up the proof into several lemmas.

Lemma 3.3. Let $O \in \mathcal{H}_{\mathbb{F}}^{n}$ be a fixed reference point, $\xi \in \partial \mathcal{H}_{\mathbb{F}}^{n}$ a parabolic fixed point and let $x \in \mathcal{H}_{\mathbb{F}}^{n}$ be a point on the geodesic from $O$ approaching $\xi$. Then we have

$$
\mu_{x}\left(L_{\Gamma}\right) \sim \frac{\sum_{\gamma \in \Gamma_{\xi}} e^{-\delta_{\Gamma} d\left(x, \gamma^{-1} x\right)}}{\sum_{\gamma \in \Gamma_{\xi}} e^{-\delta_{\Gamma} d\left(O, \gamma^{-1} O\right)}} e^{-\delta_{\Gamma} d(O, x)} .
$$

Proof. Choose a fundamental domain $F$ for the stabilizer $\Gamma_{\xi}$ of $\xi$ in such a way that $F$ is "vertically homogeneous" (that is the sides of $F$ are hyperplanes perpendicular to the hyperplane $\left\{x_{1}=0\right\}$ ). If $B$ is a horoball whose base point is $\xi$, then $F \cap B$ is a fundamental domain for $\Gamma$ in $\hat{C}$ and hence, if $y \notin \hat{C}$, we have that $\Gamma \cdot y \cap(F \cap B)=\emptyset$. Fix a point $O \in F \cap B$ and let $\sigma:[0, \infty) \rightarrow \mathcal{H}_{\mathbb{F}}^{n}$ be a geodesic ray starting at $O$ and approaching $\xi$. If $x=\sigma\left(t_{0}\right)$ for some $t_{0} \in(0, \infty)$, by definition of $\mu_{x}$ and manipulating in an obvious way the sum in the Poincaré series, we have that

$$
\mu_{x}\left(L_{\Gamma}\right)=\lim _{s \rightarrow \delta_{\Gamma}^{+}} \mu_{s, x}(\Gamma x)=\lim _{s \rightarrow \delta_{\Gamma}^{+}} \frac{P_{s}(x, y)}{P_{s}(O, y)}=\lim _{s \rightarrow \delta_{\Gamma}^{+}} \frac{\sum_{\gamma \in \Gamma_{\xi}} \sum_{\gamma^{\prime} y \in F} e^{-s d\left(x, \gamma \gamma^{\prime} y\right)}}{\sum_{\gamma \in \Gamma_{\xi}} \sum_{\gamma^{\prime} y \in F} e^{-s d\left(O, \gamma \gamma^{\prime} y\right)}} .
$$

To estimate the above quotient we are going to use repeatedly the inequalities proven in Lemma 3.1 applied to the geodesic triangles with vertices $x, O$ and $\gamma^{\prime} y$ and with vertices $\gamma^{-1} x, x, \gamma^{\prime} y$, where $\gamma^{\prime} \in \Gamma, \gamma \in \Gamma_{\xi}$ and $y$ is chosen to be in the complement of $\hat{C}$. Our choice of $y$ is determined by the fact that we want to insure that both the angle between $\sigma$ and the geodesic ray connecting $\gamma^{\prime} y$ and $O$ and the angle between the geodesic ray connecting $\gamma^{\prime} y$ and $x$ and the geodesic ray connecting $x$ and $\gamma^{-1} x$ are bounded away from zero. See Figure 1.

From Lemma 3.1 applied to the geodesic triangle with vertices at the points $\gamma^{-1} x, x, \gamma^{\prime} y$, we have that there exists a constant $C_{1}$, independent of $\gamma \in \Gamma_{\xi}, x \in \sigma$ and $\gamma^{\prime} y \in F$, such that

$$
d\left(x, \gamma^{-1} x\right)-C_{1} \leq d\left(\gamma^{-1} x, \gamma^{\prime} y\right)-d\left(x, \gamma^{\prime} y\right) \leq d\left(x, \gamma^{-1} x\right) .
$$




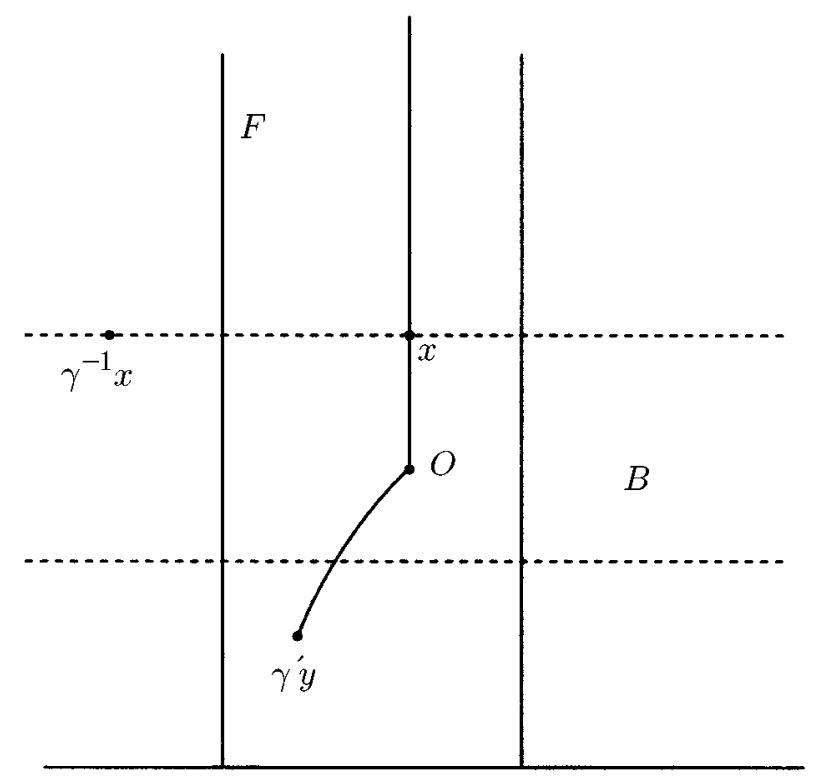

Figure 1

Thus we have

$$
\begin{aligned}
P_{s}(x, y) & =\sum_{\gamma \in \Gamma_{\xi}} \sum_{\gamma^{\prime} y \in F} e^{-s d\left(x, \gamma \gamma^{\prime} y\right)}=\sum_{\gamma \in \Gamma_{\xi}} \sum_{\gamma^{\prime} y \in F} e^{-s d\left(\gamma^{-1} x, \gamma^{\prime} y\right)} \\
& =\sum_{\gamma \in \Gamma_{\xi}} \sum_{\gamma^{\prime} y \in F} e^{-s d\left(x, \gamma^{\prime} y\right)} e^{-s\left[d\left(\gamma^{-1} x, \gamma^{\prime} y\right)-d\left(x, \gamma^{\prime} y\right)\right]} \\
& \sim \sum_{\gamma \in \Gamma_{\xi}} e^{-s d\left(x, \gamma^{-1} x\right)} \sum_{\gamma^{\prime} y \in F} e^{-s d\left(x, \gamma^{\prime} y\right)}
\end{aligned}
$$

where by the notation $a \sim b$ we mean that there exist constants $k^{\prime}$ and $k^{\prime \prime}$ such that $k^{\prime} a \leq b \leq k^{\prime \prime} a$.

Again from Lemma 3.1, applied this time to the geodesic triangle with vertices at the points $O, x, \gamma^{\prime} y$, we have that there exists a constant $C_{2}$, independent of $x \in \sigma, \gamma^{\prime} y \in F$, such that

$$
d(O, x)-C_{2} \leq d\left(x, \gamma^{\prime} y\right)-d\left(O, \gamma^{\prime} y\right) \leq d(O, x) .
$$

Hence, from above,

$$
\begin{aligned}
P_{s}(x, y) & \sim \sum_{\gamma \in \Gamma_{\xi}} e^{-s d\left(x, \gamma^{-1} x\right)} \sum_{\gamma^{\prime} y \in F} e^{-s d\left(x, \gamma^{\prime} y\right)} \\
& =\sum_{\gamma \in \Gamma_{\xi}} e^{-s d\left(x, \gamma^{-1} x\right)} \sum_{\gamma^{\prime} y \in F} e^{-s d\left(O, \gamma^{\prime} y\right)} e^{-s\left[d\left(x, \gamma^{\prime} y\right)-d\left(O \gamma^{\prime} y\right)\right]} \\
& \sim \sum_{\gamma \in \Gamma_{\xi}} e^{-s d\left(x, \gamma^{-1} x\right)} e^{-s d(O, x)} \sum_{\gamma^{\prime} y \in F} e^{-s d\left(O, \gamma^{\prime} y\right)} .
\end{aligned}
$$


Since the above estimates are all independent of the point $x \in \sigma$, we have that

$$
P_{s}(O, y) \sim \sum_{\gamma \in \Gamma_{\xi}} e^{-s d\left(O, \gamma^{-1} O\right)} \sum_{\gamma^{\prime} y \in F} e^{-s d\left(O, \gamma^{\prime} y\right)},
$$

which implies that

$$
\frac{P_{s}(x, y)}{P_{s}(O, y)} \sim \frac{\sum_{\gamma \in \Gamma_{\xi}} e^{-s d\left(x, \gamma^{-1} x\right)}}{\sum_{\gamma \in \Gamma_{\xi}} e^{-s d\left(O, \gamma^{-1} O\right)}} e^{-s d(O, x)} .
$$

Let us observe now that $\sum_{\gamma \in \Gamma_{\xi}} e^{-\delta_{\Gamma} d\left(O, \gamma^{-1} O\right)}<\infty$. In fact, because of the properties of the Patterson-Sullivan measure, we have that for any $\eta \in \partial \mathcal{H}_{\mathbb{F}}^{n}$ and $\gamma \in \Gamma_{\xi}$

$$
\begin{aligned}
\mu_{O}\left(\gamma^{-1} \bar{F} \cap \partial \mathcal{H}_{\mathbb{F}}^{n}\right) & =e^{-\delta_{\Gamma}\left(b_{\eta}(O)-b_{\eta}\left(\gamma^{-1} O\right)\right)} \mu_{\gamma^{-1} O}\left(\gamma^{-1} \bar{F} \cap \partial \mathcal{H}_{\mathbb{F}}^{n}\right) \\
& =e^{-\delta_{\Gamma}\left(b_{\eta}(O)-b_{\eta}\left(\gamma^{-1} O\right)\right)} \gamma_{*}^{-1} \mu_{O}\left(\gamma^{-1} \bar{F} \cap \partial \mathcal{H}_{\mathbb{F}}^{n}\right) \\
& =e^{-\delta_{\Gamma}\left(b_{\eta}(O)-b_{\eta}\left(\gamma^{-1} O\right)\right)} \mu_{O}\left(\bar{F} \cap \partial \mathcal{H}_{\mathbb{F}}^{n}\right),
\end{aligned}
$$

so that

$$
\begin{aligned}
\infty & >\sum_{\gamma \in \Gamma_{\xi}} \mu_{O}\left(\gamma^{-1} \bar{F} \cap \partial \mathcal{H}_{\mathbb{F}}^{n}\right)=\sum_{\gamma \in \Gamma_{\xi}} e^{-\delta_{\Gamma}\left(b_{\eta}(O)-b_{\eta}\left(\gamma^{-1} O\right)\right)} \mu_{O}\left(\bar{F} \cap \partial \mathcal{H}_{\mathbb{F}}^{n}\right) \\
& =\mu_{O}\left(\bar{F} \cap \partial \mathcal{H}_{\mathbb{F}}^{n}\right) \sum_{\gamma \in \Gamma_{\xi}} e^{-\delta_{\Gamma} d\left(O, \gamma^{-1} O\right)}
\end{aligned}
$$

as we claimed.

Then from (3.2) we have that

$$
\begin{aligned}
\mu_{x}\left(L_{\Gamma}\right) & =\lim _{s \rightarrow \delta_{\Gamma}^{+}} \frac{P_{s}(x, y)}{P_{s}(O, y)} \sim \lim _{s \rightarrow \delta_{\Gamma}^{+}} \frac{\sum_{\gamma \in \Gamma_{\xi}} e^{-s d\left(x, \gamma^{-1} x\right)}}{\sum_{\gamma \in \Gamma_{\xi}} e^{-s d\left(O, \gamma^{-1} O\right)}} e^{-s d(O, x)} \\
& =\frac{\sum_{\gamma \in \Gamma_{\xi}} e^{-\delta_{\Gamma} d\left(x, \gamma^{-1} x\right)}}{\sum_{\gamma \in \Gamma_{\xi}} e^{-\delta_{\Gamma} d\left(O, \gamma^{-1} O\right)}} e^{-\delta_{\Gamma} d(O, x)}
\end{aligned}
$$

which completes the proof.

Continuation of the proof of Theorem 3.2. To simplify the notation, set $z=e^{d(O, x)}$ and $h_{x}(g)=e^{-d\left(x, g^{-1} x\right)}$ for $g \in G$ so that (3.1) can be rewritten as

$$
\mu_{x}\left(L_{\Gamma}\right) \sim \frac{\sum_{\gamma \in \Gamma_{\xi}} h_{x}^{\delta_{\Gamma}}(\gamma)}{\sum_{\gamma \in \Gamma_{\xi}} h_{O}^{\delta_{\Gamma}}(\gamma)} z^{-\delta_{\Gamma}} .
$$

Note that, given any parabolic fixed point $\xi$, we have that $\Gamma_{\xi} \subset M \ltimes N$ and we would like to be able to say, for what follows, that the Zariski closure of $\Gamma_{\xi}$ is a nilpotent subgroup of $N$, which unfortunately is not quite true. But since we are actually only going to look at the $\Gamma_{\xi}$-orbits of $x$ and $O$, the following lemma, whose proof we postpone for a while, will solve this problem.

Lemma 3.4. Let $N$ be a linear connected simply connected nilpotent group, $M$ a compact group of automorphisms of $N$ and $\Gamma \subset Q=M \ltimes N$ an almost nilpotent discrete subgroup. Then there exist subgroups $\Gamma_{1} \subset \Gamma$ of finite index and $\Gamma_{2} \subset N$ discrete such that $\Gamma_{1}$ is isomorphic to $\Gamma_{2}$ and there exist $\Gamma_{1}$-orbits in $N$ which are $\Gamma_{2}$-orbits. 
We want to point out, in order to apply this result, that it is easy to check that if $\Gamma_{1} \cdot x=\Gamma_{2} \cdot x$, then also $\Gamma_{1} \cdot O=\Gamma_{2} \cdot O$ (because the translation $\tau_{x}$ from $O$ to $x$ along the geodesic $\sigma$ is an element of $A$ ). Then, applying Lemma 3.4 with $\Gamma_{\xi}=\Gamma$ and letting $H$ be the Zariski closure of $\Gamma_{2}$, we have that $H$ is a nilpotent group in which $\Gamma_{2}$ is cocompact and can be written as an extension $0 \rightarrow \mathbb{R}^{l} \rightarrow H \rightarrow \mathbb{R}^{k} \rightarrow 0$, where $l=\operatorname{dim}(Z(H) \cap \operatorname{Im} \mathbb{F})$ and $k=\operatorname{dim}(H /(Z(H) \cap \operatorname{Im} \mathbb{F}))$, as we wanted.

We now claim the following:

Lemma 3.5. Let $\xi, O, x$ be as in Lemma 3.3. Then $\delta_{\Gamma}>\frac{2 l+k}{2}$ and

$$
\mu_{x}\left(L_{\Gamma}\right)<C e^{\frac{2 l+k}{2} d(O, x)} .
$$

This will immediately complete the proof of Theorem 3.2. In fact, if we now assume that $\mu_{x}$ has an atom at $\xi \in \mathcal{H}_{\mathbb{F}}^{n}$, then, just from the conformal properties of the Patterson-Sullivan measure we have that

$$
\begin{aligned}
\mu_{x}\left(L_{\Gamma}\right) & =\int_{\partial \mathcal{H}_{\mathbb{F}}^{n}} d \mu_{x}(\eta)=\int_{\partial \mathcal{H}_{\mathbb{F}}^{n}} e^{\delta_{\Gamma}\left(b_{\eta}(O)-b_{\eta}(x)\right)} d \mu_{O}(\eta) \\
& \geq e^{\delta_{\Gamma}\left(b_{\xi}(O)-b_{\xi}(x)\right)} \mu_{O}(\xi)=C^{\prime} e^{\delta_{\Gamma} d(O, x)}
\end{aligned}
$$

where $O$ is a fixed reference point on the geodesic connecting $x$ to $\xi$ and $C^{\prime}=$ $\mu_{O}(\infty)$. But this, together with (3.4), contradicts our previous estimate of $\delta_{\Gamma}$ in Lemma 3.5.

Proof of Lemma 3.5. To prove (3.4) we first claim that $\sum_{\gamma \in \Gamma_{\xi}} h_{O}^{\delta_{\Gamma}}(\gamma)$ converges if and only if $\delta_{\Gamma}>\frac{2 l+k}{2}$ or equivalently, because $\Gamma_{\xi}$ is cocompact in $H$, that if $\lambda$ is the volume form induced on $H$ by the ambient Riemannian metric, then $\int_{H} h_{O}^{\delta_{\Gamma}}(g) d \lambda_{O}(g)$ converges if and only if $\delta_{\Gamma}>\frac{2 l+k}{2}$. This follows directly from [He2, Chapter IX, Theorem 3.8] and the proof of [He1, Theorem 6.4, p. 437], but for $\mathbb{F}=\mathbb{R}, \mathbb{C}$ or $\mathbb{H}$ the computation is very elementary and we sketch it here for the sake of completeness. The first step will be to show that for $g=(v, a) \in N$ (and hence in particular for $g \in H$ )

$$
h_{O}(g) \sim \frac{1}{\left(1+|v|^{4}+|a|^{2}\right)^{1 / 2}} .
$$

In fact, observing that $N$ is the nilradical of the stabilizer of the line $\mathbb{F}(1,0, \ldots, 0,1)$, we can represent $N$ by

$$
N=\left\{\left(\begin{array}{ccc}
1-a-\frac{v^{*} v}{2} & v^{*} & a+\frac{v^{*} v}{2} \\
-v & \text { Id } & v \\
-a-\frac{v^{*} v}{2} & v^{*} & 1+a+\frac{v^{*} v}{2}
\end{array}\right) \mid v \in \mathbb{F}^{n-1}, a \in \operatorname{Im} \mathbb{F}\right\},
$$

where $v^{*}$ is the transpose conjugate of $v$. Then, looking at the $N$-orbit of $w=$ $(0, \ldots, 0,1)$ and projectivizing the result, one obtains that

$$
d(w,(v, a) w)=\cosh ^{-1}\left(1-\frac{\left|a+\frac{v^{*} v}{2}\right|+|v|^{2}}{\left|1+a+\frac{v^{*} v}{2}\right|^{2}}\right)^{-\frac{1}{2}},
$$

where $d$ is the hyperbolic distance on $\mathcal{H}_{\mathbb{F}}^{n}$. Then (3.5) will follow just by formally manipulating the above equation and using the fact that $\cosh ^{-1} t \sim \log 2 t$. 
Then the integral $\int_{H} \frac{d \lambda_{O}(g)}{\left(1+|v|^{4}+|a|^{2}\right)^{\delta_{\Gamma} / 2}}$ can be evaluated by elementary methods. In fact, if $|a|=x$ and $|v|=y$, then

$$
\begin{aligned}
\int_{H} \frac{d \lambda_{O}(g)}{\left(1+|v|^{4}+|a|^{2}\right)^{\delta_{\Gamma} / 2}} & =\int_{\mathbb{R}} \int_{\mathbb{R}} \frac{x^{l-1} y^{k-1} d x d y}{\left(1+x^{2}+y^{4}\right)^{\delta_{\Gamma} / 2}} \\
& =\int_{\mathbb{R}} \frac{y^{k-1} d y}{\left(1+y^{4}\right)^{\delta_{\Gamma} / 2-l / 2}} \int_{\frac{-\pi}{2}}^{\frac{\pi}{2}} \frac{\tan ^{l-1} \theta d \theta}{\cos ^{2} \theta\left(1+\tan ^{2} \theta\right)^{\delta_{\Gamma} / 2}},
\end{aligned}
$$

where we set $x=\tan \theta\left(1+y^{4}\right)^{1 / 2}$. It is now an exercise in calculus to show that the above integral converges if and only if $\delta_{\Gamma}>\frac{2 l+k}{2}$, hence deducing that we have convergence of the sum $\sum_{\gamma \in \Gamma_{\xi}} h_{O}^{\delta_{\Gamma}}(\gamma)$ if and only if $\delta_{\Gamma}>\frac{2 l+k}{2}$.

To complete the proof, let us denote by $\tau_{x} \in A$ the translation from $O$ to $x$ along the geodesic $\sigma$. This acts on $H$ and we have that

$$
d \lambda_{x}=\tau_{x} \lambda_{O}=e^{d(O, x)(2 l+k)} d \lambda_{O}=z^{2 l+k} d \lambda_{O},
$$

so that we can rewrite $(3.3)$ as

$$
\begin{aligned}
& \mu_{x}\left(L_{\Gamma}\right) \sim \frac{\sum_{\gamma \in \Gamma_{\xi}} h_{x}^{\delta_{\Gamma}}(\gamma)}{\sum_{\gamma \in \Gamma_{\xi}} h_{O}^{\delta_{\Gamma}}(\gamma)} z^{-\delta_{\Gamma}} \sim \frac{\int_{H} h_{x}^{\delta_{\Gamma}}(g) d \lambda_{x}(g)}{\int_{H} h_{O}^{\delta_{\Gamma}}(g) d \lambda_{O}(g)} z^{-\delta_{\Gamma}} \\
& =\frac{\int_{H} h_{x}^{\delta_{\Gamma}}(g) \tau_{x} d \lambda_{O}(g)}{\int_{H} h_{O}^{\delta_{\Gamma}}(g) d \lambda_{O}(g)} z^{-\delta_{\Gamma}}=\frac{\int_{H}\left(h_{x}^{\delta_{\Gamma}} \circ \tau_{x}^{-1}\right)(g) e^{d(O, x)(2 l+k)} d \lambda_{O}(g)}{\int_{H} h_{O}^{\delta_{\Gamma}}(g) d \lambda_{O}(g)} z^{-\delta_{\Gamma}} \\
& =\frac{\int_{H} h_{O}^{\delta_{\Gamma}}(g) z^{2 l+k} d \lambda_{O}(g)}{\int_{H} h_{O}^{\delta_{\Gamma}}(g) d \lambda_{O}(g)} z^{-\delta_{\Gamma}}=z^{2 l+k-\delta_{\Gamma}}
\end{aligned}
$$

which implies, with the lower bound found for $\delta_{\Gamma}$, that

$$
\mu_{x}\left(L_{\Gamma}\right) \leq C z^{2 l+k-\delta_{\Gamma}}<C z^{\frac{2 l+k}{2}}
$$

where $C$ is a constant.

One more piece of unfinished business:

Proof of Lemma 3.4. Cf. [Au]. Since $\Gamma$ is almost nilpotent, choose $\Gamma_{1}^{*} \subset \Gamma$ of finite index and nilpotent. Let $H$ be the Zariski closure of $\Gamma_{1}^{*}$, so that $H$ is nilpotent with finitely many components. Let $H^{\circ}$ be the connected component of the identity in $H$ and let $\Gamma_{1}^{* *}=H^{\circ} \cap \Gamma_{1}^{*}$. If we denote the projection by $\pi: Q \rightarrow M$, we have that $\overline{\pi\left(H^{\circ}\right)}$ is nilpotent, connected and contained in a compact, and hence is a torus $T$. It follows that $H^{\circ} \subset T \ltimes N$ (and $\mathfrak{h} \subset \mathfrak{t} \ltimes \mathfrak{n}$ ). Choose $g \in H^{\circ}$ such that the closure of the group generated by $\pi(g)$ is $T$. By [W, Lemma 5.2], we can choose a semidirect product decomposition of $Q$ such that $g=(m, n) \in M \ltimes N$ satisfies $(m, 1)(1, n)=(1, n)(m, 1)$ (that is there exists $\eta=\left(1, n_{1}\right) \in M \ltimes N$ such that $\eta g \eta^{-1}=(m, n)$ has the above property). Observe that $\operatorname{Ad}(m, n)=\operatorname{Ad}(m, 1) \operatorname{Ad}(1, n)$ is the $\operatorname{Jordan}$ decomposition because $\operatorname{Ad}(m, 1)$ is semisimple, $\operatorname{Ad}(1, n)$ is nilpotent and they commute. Moreover, the Lie algebra $\mathfrak{h}=\operatorname{Lie}\left(H^{\circ}\right)$ of $H$ is invariant under $\operatorname{Ad}(m, 1)$ and $\operatorname{Ad}(1, n)$, since it is $\operatorname{Ad}(m, n)$ invariant, so that $\left.\operatorname{Ad}(m, n)\right|_{\mathfrak{h}}=\left.\left.\operatorname{Ad}(m, 1)\right|_{\mathfrak{h}} \operatorname{Ad}(1, n)\right|_{\mathfrak{h}}$. But $\mathfrak{h}$ is nilpotent, hence $\left.\operatorname{Ad}(m, n)\right|_{\mathfrak{h}}$ is unipotent, which implies that $\operatorname{Ad}(m, 1)$ acts trivially on $\mathfrak{h}$. Since $\pi(g)=m$ is a topological generator of $T, T$ acts trivially on $\mathfrak{h}$ as well. Hence $\mathfrak{h} \subset \operatorname{Lie}(Q)^{T}=\mathfrak{t} \ltimes \mathfrak{n}^{T}$, where the last equality follows from the fact that $\mathfrak{t}$ is Abelian. Let $W \subset N$ be the subgroup fixed by $T$ so that $\Gamma_{1}^{* *} \subset H^{\circ} \subset T \ltimes W \subset M \ltimes N$. To 
conclude the proof it will be enough to define $\Gamma_{1}$ to be a torsion-free subgroup of finite index in $\Gamma_{1}^{* *}$ which projects isomorphically onto a subgroup $\Gamma_{2} \subset W$ (notice that the kernel of such projection is at worst finite, so we may eliminate it by taking an appropriate subgroup). The assertion about the orbits follows from the fact that $T$ acts trivially on $W$ and hence $\Gamma_{1} \cdot w=\Gamma_{2} \cdot w$ for any $w \in W$.

With a last bit of work we can now conclude:

Theorem 3.6. The Patterson-Sullivan measure $\mu_{x}$ has no atoms.

Proof. Let us assume that $\mu_{x}$ has an atom at $\xi \in L_{\Gamma}$. If $\Gamma_{\xi}$ is the stabilizer of $\xi$ in $\Gamma$ and $\gamma \in \Gamma_{\xi}$, then, by the properties of $\mu_{x}$, we have that $\mu_{\gamma x}(\xi)=\gamma_{*} \mu_{x}(\xi)=$ $\mu_{x}\left(\gamma^{-1} \xi\right)=\mu_{x}(\xi)$. Again because of the transformation properties of $\mu_{x}$ relative to $\mu_{y}$, for $x, y \in \mathcal{H}_{\mathbb{F}}^{n}$, we have that $\mu_{x}(\xi)=e^{\delta_{\Gamma}\left(b_{\xi}(\gamma x)-b_{\xi}(x)\right)} \mu_{\gamma x}(\xi)$, which, together with the above equality implies that $b_{\xi}(\gamma x)=b_{\xi}(x)$. Since horospheres are level sets of Busemann functions, it follows that $\Gamma_{\xi}$ is contained in the stabilizer of a horosphere based at $\xi$, that is $\Gamma_{\xi} \subset M N \subset M A N$. Hence, either $\Gamma_{\xi}$ is trivial or $\xi$ is a parabolic fixed point. We shall argue now that $\Gamma_{\xi}$ cannot be trivial, hence implying that all atoms would have to be parabolic. But, by Theorem 3.2, we know that this is impossible, which completes the proof. Thus, let us assume that $\Gamma_{\xi}$ is trivial. Then we have

$$
\begin{aligned}
\mu_{x}(\Gamma \cdot \xi) & =\sum_{\gamma \in \Gamma} \mu_{x}\left(\gamma^{-1} \xi\right)=\sum_{\gamma \in \Gamma} \gamma_{*} \mu_{x}(\xi)=\sum_{\gamma \in \Gamma} \mu_{\gamma x}(\xi) \\
& =\sum_{\gamma \in \Gamma} e^{-\delta_{\Gamma}\left(b_{\xi}(\gamma x)-b_{\xi}(x)\right)} \mu_{x}(\xi)=\mu_{x}(\xi) \sum_{\gamma \in \Gamma} e^{\delta_{\Gamma}\left(b_{\xi}(\gamma x)-b_{\xi}(x)\right)} \\
& \geq \mu_{x}(\xi) \sum_{\gamma \in \Gamma} e^{-\delta_{\Gamma} d(x, \gamma x)}
\end{aligned}
$$

where the inequality follows from the above mentioned property of the Busemann functions. Hence

$$
\sum_{\gamma \in \Gamma} e^{-\delta_{\Gamma} d(x, \gamma x)} \leq \frac{\mu_{x}(\Gamma \cdot \xi)}{\mu_{x}(\xi)} \leq C \mu_{x}\left(L_{\Gamma}\right)<\infty
$$

and the following result will provide the required contradiction.

Proposition 3.7. $\sum_{\gamma \in \Gamma} e^{-\delta_{\Gamma} d(x, \gamma x)}$ diverges.

Proof. Let us write $\Gamma=\left\{\gamma_{n}: n \in \mathbb{Z}\right\}$. If $\sum_{\gamma \in \Gamma} e^{-\delta_{\Gamma} d(x, \gamma x)}<\infty$ then, given $\epsilon>0$, there exists $N>0$ such that $\sum_{n>N} e^{-\delta_{\Gamma} d\left(x, \gamma_{n} x\right)}<\epsilon$. If $\xi_{j} \in \partial \mathcal{H}_{\mathbb{F}}^{n}$ is the endpoint of the geodesic joining $x$ and $\gamma_{j} x$, denote by $B_{j}^{C} \subset \partial \mathcal{H}_{\mathbb{F}}^{n}$ the sub-Riemannian ball of radius $r_{j}=C e^{-d\left(x, \gamma_{j} x\right)}$ centered at $\xi_{j}$. In [C, Theorem 5.4] it is proven that if $\mu_{x}$ does not consist of a single atom (which is our case because we are assuming that $\Gamma$ is not elementary), there exist constants $C_{1}, C_{2}$ independent of $\gamma_{j}$ such that

$$
C_{1} \leq \frac{\mu_{x}\left(B_{j}^{C}\right)}{r_{j}^{\delta_{\Gamma}}} \leq C_{2}
$$

(actually, the proof of [C, Theorem 5.4] applies to the case of geometrically cocompact groups; however, exactly the same argument as in the first three paragraphs 


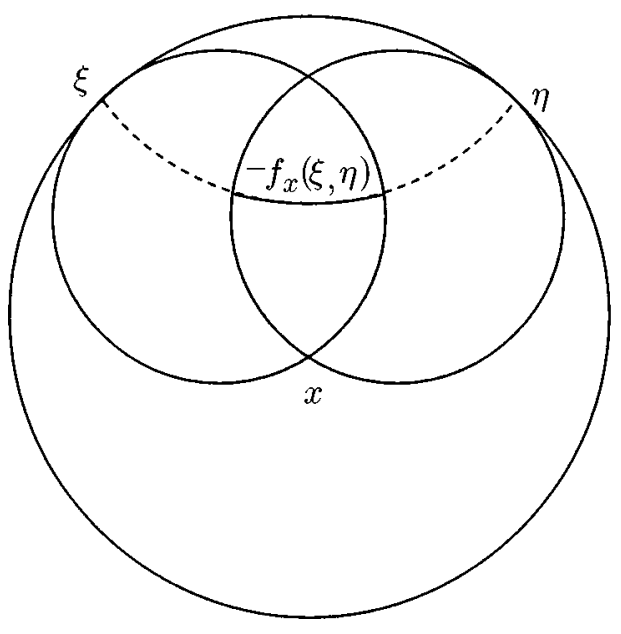

Figure 2

of this proof shows the above mentioned result). Hence we have

$$
\mu_{x}\left(\bigcup_{n>N} B_{n}^{C}\right) \leq \sum_{n>N} \mu_{x}\left(B_{n}^{C}\right) \leq C_{2} C^{\delta_{\Gamma}} \sum_{n>N} e^{-\delta_{\Gamma} d\left(x, \gamma_{n} x\right)}<C_{2} C^{\delta_{\Gamma}} \epsilon .
$$

Since $L_{\Gamma}^{\mathrm{rad}} \subseteq \bigcup_{C>0} \bigcap_{N \geq 1} \bigcup_{n>N} B_{n}^{C}$ and the above estimate holds for any $\epsilon>0$, it follows that $\mu_{x}\left(L_{\Gamma}^{\mathrm{rad}}\right)=0$, which is impossible by Theorem 3.2.

\section{4. $\delta_{\Gamma}$-FINITENESS OF VOLUME}

The goal of this section will be to define a measure $\nu$ on $T / \Gamma$ (where $T$ is the unit tangent bundle to $\mathcal{H}_{\mathbb{F}}^{n}$ ) which is invariant with respect to the quotient geodesic flow and has finite volume. Although the construction of such measure (but not its finiteness) is known even in the more general case of CAT(-1)-spaces (see [Bw] or $[\mathrm{K}]$ ), we recall it here. In order to define the measure $\nu$ it will be enough to construct a measure $\tilde{\nu}$ on $T$ which is invariant both under $\Gamma$ and under the geodesic flow. To achieve this, recall that every $v_{x} \in T$, where $x \in \mathcal{H}_{\mathbb{F}}^{n}$, can be identified with the geodesic $\sigma$ such that $\sigma(0)=x$ and $\sigma^{\prime}(0)=v_{x}$. This implies that, as usual, we can fiber $T$ over $\partial \mathcal{H}_{\mathbb{F}}^{n} \times \partial \mathcal{H}_{\mathbb{F}}^{n} \backslash\{$ diagonal $\}$ by geodesic lines and, using the notation $\partial \mathcal{H}_{\mathbb{F}}^{n} \times \partial \mathcal{H}_{\mathbb{F}}^{n} \backslash\{$ diagonal $\}=\left(\partial \mathcal{H}_{\mathbb{F}}^{n}\right)_{0}^{2}$, that a $\Gamma$-invariant measure on $\left(\partial \mathcal{H}_{\mathbb{F}}^{n}\right)_{0}^{2}$ combined with the arclength along geodesics is going to give a $\Gamma$-invariant and geodesic flow invariant measure $\tilde{\nu}$ on $T$. If $\delta_{\Gamma}$ is the critical exponent of $\Gamma$, let us define a family of measures $m_{x}$ on $\left(\partial \mathcal{H}_{\mathbb{F}}^{n}\right)_{0}^{2}$, parameterized by $x \in \mathcal{H}_{\mathbb{F}}^{n}$, by

$$
m_{x}(\xi, \eta)=h_{x}(\xi, \eta) \mu_{x}(\xi) \mu_{x}(\eta)
$$

where

$$
h_{x}(\xi, \eta)=\lim _{u \rightarrow \xi, v \rightarrow \eta} e^{\delta_{\Gamma}(d(u, x)+d(x, v)-d(u, v))}=e^{-\delta_{\Gamma} f_{x}(\xi, \eta)},
$$

with

$$
f_{x}(\xi, \eta)=\lim _{u \rightarrow \xi, v \rightarrow \eta}(d(u, v)-d(u, x)-d(x, v)) .
$$

See Figure 2. 
With this notation we can write

$$
m_{x}(\xi, \eta)=\frac{\mu_{x}(\xi) \times \mu_{x}(\eta)}{e^{\delta_{\Gamma} f_{x}(\xi, \eta)}}
$$

and we are going to show that the family of measures $m_{x}$ is independent of the parameter $x$, hence defining a single measure $m$ on $\left(\partial \mathcal{H}_{\mathbb{F}}^{n}\right)_{0}^{2}$, which we shall then prove is $\Gamma$-invariant. To check the independence of $x$, it is just a matter of chasing the definitions: in fact, if $x, y \in \mathcal{H}_{\mathbb{F}}^{n}$, we have that

$$
\frac{\mu_{x}(\xi) \times \mu_{x}(\eta)}{e^{\delta_{\Gamma} f_{x}(\xi, \eta)}}=\frac{\mu_{y}(\xi) \times \mu_{y}(\eta)}{e^{\delta_{\Gamma} f_{y}(\xi, \eta)}}
$$

provided that

$$
\frac{1}{e^{\delta_{\Gamma} f_{x}(\xi, \eta)}}=\frac{e^{\delta_{\Gamma}\left(b_{\xi}(x)-b_{\xi}(y)+b_{\eta}(x)-b_{\eta}(y)\right)}}{e^{\delta_{\Gamma} f_{y}(\xi, \eta)}}
$$

or, in other words, that

$$
f_{x}(\xi, \eta)-f_{y}(\xi, \eta)+b_{\xi}(x)-b_{\xi}(y)+b_{\eta}(x)-b_{\eta}(y)=0,
$$

which follows immediately from the definition of $f_{x}$ and the fact that $b_{\xi}(x)-b_{\xi}(y)=$ $\lim _{u \rightarrow \xi}(d(x, u)-d(y, u))$.

The $\Gamma$-invariance of $m=m_{x}$ will be proven if we show that for any $\gamma \in \Gamma$, $\gamma^{*} m=g^{*} m_{x}=m_{\gamma x}=m$. This follows immediately from the corresponding property for $\mu_{x}$ and from the fact that $\Gamma$ acts by isometries, hence insuring that $f_{x}\left(\gamma^{-1} \xi, \gamma^{-1} \eta\right)=f_{\gamma x}(\xi, \eta)$. In fact,

$$
\begin{aligned}
\gamma^{*} m_{x}(\xi, \eta) & =m_{x}\left(\gamma^{-1} \xi, \gamma^{-1} \eta\right)=\frac{\mu_{x}\left(\gamma^{-1} \xi\right) \times \mu_{x}\left(\gamma^{-1} \eta\right)}{e^{\delta_{\Gamma} f_{x}\left(\gamma^{-1} \xi, \gamma^{-1} \eta\right)}} \\
& =\frac{\mu_{\gamma x}(\xi) \times \mu_{\gamma x}(\eta)}{e^{\delta_{\Gamma} f_{\gamma x}(\xi, \eta)}}=m_{\gamma x}(\xi, \eta) .
\end{aligned}
$$

Now we can prove that the volume of $T / \Gamma$ is $\delta_{\Gamma}$-finite.

Theorem 4.1. $\nu(T / \Gamma)<\infty$.

We start with the following lemma, whose proof we shall postpone for a while.

Lemma 4.2. Let $\varphi: \mathcal{H}_{\mathbb{F}}^{n} \rightarrow[0, \infty)$ be defined by $\varphi(x)=\mu_{x}\left(L_{\Gamma}\right)$. Then, for $\epsilon>0$ we have that $\varphi \in L^{2}\left(\operatorname{Hull}^{\epsilon}\left(L_{\Gamma}\right) / \Gamma, \lambda\right)$, where $\operatorname{Hull}^{\epsilon}\left(L_{\Gamma}\right)$ is an $\epsilon$-neighborhood of the convex hull Hull $\left(L_{\Gamma}\right)$ of the limit set and $\lambda$ is the volume form induced by the Riemannian metric.

Proof of Theorem 4.1. Let $Z \rightarrow T$ be a fiber bundle whose fiber over a point $v_{x} \in T$ is the image under the exponential map of the normal space to the geodesic $\gamma$ such that $\gamma(0)=x$ and $\gamma^{\prime}(0)=v_{x}$. Then $Z$ can be identified with $\mathcal{H}_{\mathbb{F}}^{n} \times\left(\partial \mathcal{H}_{\mathbb{F}}^{n}\right)_{0}^{2}$. In fact, on the one hand we have a map $p: Z \rightarrow \mathcal{H}_{\mathbb{F}}^{n}$ obtained by taking the inclusion of the fibers of $Z \rightarrow T$ into $\mathcal{H}_{\mathbb{F}}^{n}$ in the canonical way; for such a map $p$ the fibers can be easily identified with the space of geodesics $\left(\partial \mathcal{H}_{\mathbb{F}}^{n}\right)_{0}^{2}$. On the other hand we also have a map $Z \rightarrow\left(\partial \mathcal{H}_{\mathbb{F}}^{n}\right)_{0}^{2}$ obtained by mapping $Z$ onto $T$ and then mapping a point $v_{x} \in T$ to the corresponding geodesic in $\left(\partial \mathcal{H}_{\mathbb{F}}^{n}\right)_{0}^{2}$. Now, on $Z=\mathcal{H}_{\mathbb{F}}^{n} \times\left(\partial \mathcal{H}_{\mathbb{F}}^{n}\right)_{0}^{2}$ we want to consider the measure $d \alpha$ defined by $d \alpha(x,(\xi, \eta))=d \lambda_{\xi, \eta}(x) \times d m(\xi, \eta)$, where $d \lambda_{\xi, \eta}(x)$ is the restriction of the volume form $d \lambda$ to a unit neighborhood of the geodesic connecting $\xi$ and $\eta$. Since $\alpha(Z / \Gamma)$ is comparable to $\nu(T / \Gamma)$, to show that $\nu(T / \Gamma)$ is finite it will be enough to show that $\alpha(Z / \Gamma)$ is finite. 
Let $\left(\partial \mathcal{H}_{\mathbb{F}}^{n}\right)_{0}^{2}(x, 1)$ be the set of geodesics which pass within unit distance from $x$. If we decompose $\alpha$ with respect to the projection $p$, we obtain that the measure $\alpha_{x}$ on $p^{-1}(x)=\{x\} \times\left(\partial \mathcal{H}_{\mathbb{F}}^{n}\right)_{0}^{2}$ is just the restriction of $m$ to $\left(\partial \mathcal{H}_{\mathbb{F}}^{n}\right)_{0}^{2}(x, 1)$. We shall prove that $\alpha_{x}\left(p^{-1}(x)\right) \leq C \varphi(x)^{2}$, where $\varphi: \mathcal{H}_{\mathbb{F}}^{n} \rightarrow[0, \infty)$ is defined by $\varphi(x)=\mu_{x}\left(L_{\Gamma}\right)$ as in Lemma 4.2. Then choosing appropriately fundamental domains $F$ for the action of $\Gamma$ on $Z$ and $F_{1}$ for the action of $\Gamma$ on $\operatorname{Hull}^{1}\left(L_{\Gamma}\right)$ (where appropriately means that $\left.\operatorname{Hull}^{1}\left(L_{\Gamma}\right) \cap p(F)=F_{1}\right)$ and observing that $\alpha_{x}\left(p^{-1}(x)\right)=0$ if $x \notin \operatorname{Hull}^{1}\left(L_{\Gamma}\right)$, we shall have

$$
\begin{aligned}
\alpha(Z / \Gamma) & =\alpha(F)=\int_{X} \alpha_{x}\left(p^{-1}(x) \cap F\right) d \lambda(x)=\int_{\operatorname{Hull}^{1}\left(L_{\Gamma}\right)} \alpha_{x}\left(p^{-1}(x) \cap F\right) d \lambda(x) \\
& =\int_{\operatorname{Hull}^{1}\left(L_{\Gamma}\right) \cap p(F)} \alpha_{x}\left(p^{-1}(x)\right) d \lambda(x) \leq C \int_{F_{1}}|\varphi(x)|^{2} d \lambda(x)<\infty .
\end{aligned}
$$

Now to see that $\alpha_{x}\left(p^{-1}(x)\right) \leq C \varphi(x)^{2}$, let $C(x)=\max \left\{e^{-\delta_{\Gamma} f_{x}(\xi, \eta)}: \quad(\xi, \eta) \in\right.$ $\left.\left(\partial \mathcal{H}_{\mathbb{F}}^{n}\right)_{0}^{2}(x, 1)\right\}<\infty$, since $f_{x}(\xi, \eta)$ is continuous on $\left(\partial \mathcal{H}_{\mathbb{F}}^{n}\right)_{0}^{2}(x, 1)$. Moreover, since $f_{g x}(g \xi, g \eta)=f_{x}(\xi, \eta)$ for all $g \in O_{\mathbb{F}}(1, n)$ and $g\left(\left(\partial \mathcal{H}_{\mathbb{F}}^{n}\right)_{0}^{2}(x, 1)\right)=\left(\partial \mathcal{H}_{\mathbb{F}}^{n}\right)_{0}^{2}(g x, 1)$, we have that $C(x)=C$ for all $x \in \mathcal{H}_{\mathbb{F}}^{n}$. Then

$$
\alpha_{x}\left(p^{-1}(x)\right)=\int_{\left(\partial \mathcal{H}_{\mathbb{R}}^{n}\right)_{0}^{2}(x, 1)} d m(\xi, \eta) \leq C \int_{\left(\partial \mathcal{H}_{\mathbb{R}}^{n}\right)_{0}^{2}(x, 1)} d \mu_{x}(\xi) \times d \mu_{x}(\eta) \leq C \varphi(x)^{2},
$$

hence completing the proof.

Proof of Lemma 4.2. Decompose $\operatorname{Hull}^{\epsilon}\left(L_{\Gamma}\right) / \Gamma$ into a compact part and a finite number of cusps $C_{1}, C_{2}, \ldots, C_{m}$. To prove the lemma it suffices to show that $\left.\varphi\right|_{C_{i}} \in L^{2}\left(C_{i}\right)$. Let $C=C_{i}$ be one of the cusps; consider a half-space model for $\mathcal{H}_{\mathbb{F}}^{n}$ in which the point at infinity is a parabolic fixed point corresponding to $C$. Then

$$
\mathcal{H}_{\mathbb{F}}^{n}=\mathbb{R} \times N=\left\{\left(t, x_{1}, \ldots, x_{p}, y_{1}, \ldots, y_{q}\right) \in \mathbb{R}^{1+p+q}\right\}
$$

with the hyperbolic metric given by

$$
d t^{2}+e^{-2 t} \sum_{i} d x_{i}^{2}+e^{-4 t} \sum_{j} d y_{j}^{2}
$$

The stabilizer of $\infty$ in $\Gamma$ is an almost nilpotent group $\Gamma_{\infty}$, which we assume to be nilpotent, passing, if necessary, to a subgroup of finite index. As in $\S 3, \Gamma_{\infty} \subset M N$, with $M$ a compact subgroup of $O_{\mathbb{F}}(1, n)$. From Lemma 3.5 we have that

$$
\varphi\left(t, x_{1}, \ldots, x_{p}, y_{1}, \ldots, y_{q}\right) \leq C e^{\frac{2 l+k}{2} t}
$$

with $l, k, C$ as defined there. Set $N_{t}=\{t\} \times N$; to prove the lemma, it suffices to show that a fundamental domain for $\Gamma_{\infty}$ acting on $\operatorname{Hull}^{\epsilon}\left(L_{\Gamma}\right) \cap N_{t}$ has volume bounded by $C e^{-(2 l+k) t}$.

Set $H_{\tau}=\left\{(t, g) \in \mathbb{R} \times N=\mathcal{H}_{\mathbb{F}}^{n} \mid t \geq \tau\right\}=\bigcup_{t \geq \tau} N_{t}$. Then $\operatorname{Hull}\left(L_{\Gamma}\right) \cap H_{0}=$ $\operatorname{Hull}\left(\left(\operatorname{Hull}\left(L_{\Gamma}\right) \cap N_{0}\right) \cup\{\infty\}\right)$, since any geodesic connecting a point in $\operatorname{Hull}\left(L_{\Gamma}\right) \cap H_{0}$ with a point in $\operatorname{Hull}\left(L_{\Gamma}\right) \cap\left(\mathcal{H}_{\mathbb{F}}^{n} \backslash H_{0}\right)$ must pass through $\operatorname{Hull}\left(L_{\Gamma}\right) \cap N_{0}$. Moreover, since it is $\Gamma_{\infty}$-cocompact, $\operatorname{Hull}\left(L_{\Gamma}\right) \cap N_{0}$ is contained in some $\epsilon_{1}$ neighborhood of any given $\Gamma_{\infty}$-orbit $\Gamma_{\infty} \cdot x$ in $N_{0}$. By Lemma 3.4, we may take $\Gamma_{\infty} \cdot x$ to be contained in an orbit of a non-trivial connected subgroup $H$ of $N$ in such a way that $\Gamma_{\infty} \cdot x=\Gamma_{\infty}^{\prime} \cdot x$, with $\Gamma_{\infty}^{\prime}$ a discrete cocompact subgroup of $H$. Thus, it suffices to show that, for any $\epsilon>0$, the volume of the fundamental domain for $\Gamma_{\infty}$ acting on $\operatorname{Hull}^{\epsilon}(H \cdot x) \cap N_{t}$ has volume comparable to $e^{-(2 l+k) t}$. 
The choice of a point $y \in H \cdot x$ determines an Iwasawa decomposition $G=$ $K A N$, so in particular it determines a group $A$ of automorphisms of $N$, which can be regarded geometrically as a group of translations along the geodesic joining $y$ to $\infty$. We may choose $y$ so that $A$ preserves $H \subset N$. Let $\sigma: \mathbb{R} \rightarrow \mathcal{H}_{\mathbb{F}}^{n}$ be the geodesic with $\sigma(0)=y$ and $\sigma(-\infty)=\infty$, and let $a(t) \in A$ be the element satisfying $a(t) y=\sigma(t)$. Then $a(t)$ maps $H \cdot y$ to $H \cdot \sigma(t)$, so it maps $\operatorname{Hull}^{\epsilon}(H \cdot y)$ to $\operatorname{Hull}^{\epsilon}(H \cdot \sigma(t))$. Furthermore, for $t_{1}>t_{2}$,

$$
H \cdot \sigma\left(t_{2}\right) \subset \operatorname{Hull}\left(H \cdot \sigma\left(t_{1}\right)\right)
$$

so

$$
\operatorname{Hull}^{\epsilon}\left(H \cdot \sigma\left(t_{2}\right)\right) \subset \operatorname{Hull}^{\epsilon}\left(H \cdot \sigma\left(t_{1}\right)\right) .
$$

Thus, as $t$ approaches $\infty$, we have a family of increasing convex sets whose union is $\operatorname{Hull}^{\epsilon}(H \cdot \sigma(\infty))$. However, the latter is an $A$-invariant set, so

$$
\operatorname{Hull}^{\epsilon}(H \cdot \sigma(\infty)) \cap N_{t}=a_{t}\left(\operatorname{Hull}^{\epsilon}(H \cdot \sigma(\infty)) \cap N_{0}\right) .
$$

We claim that $\operatorname{Hull}(H \cdot \sigma(\infty)) \cap N_{0}$ is $\Gamma_{\infty}$-cocompact. If it were not, there would be a sequence of points $x_{i} \in \operatorname{Hull}(H \cdot \sigma(\infty)) \cap N_{0}$ such that $d\left(x_{i}, H \cdot \sigma(0)\right)$ tends to infinity. But then we could choose a sequence $t_{i}$ tending to infinity such that $a\left(t_{i}\right) x_{i} \in \operatorname{Hull}(H \cdot \sigma(\infty))$ has a point of accumulation at infinity not contained in the closure of $H \cdot \sigma(\infty)$. This would contradict the results of [Ad]. Hence, $\operatorname{Hull}(H \cdot \sigma(\infty)) \cap N_{0}$ is $\Gamma_{\infty}$-cocompact, as is the closure of $\operatorname{Hull}^{\epsilon}(H \cdot \sigma(\infty)) \cap N_{0}$.

As a consequence, a fundamental domain for $\Gamma_{\infty}$ acting on $\operatorname{Hull}^{\epsilon}\left(L_{\Gamma}\right) \cap H_{\tau}$, for $\tau$ large, is contained in a set of the form

$$
\begin{aligned}
\Lambda=\left\{\left(t, x_{1}, \ldots, x_{p}, y_{1}, \ldots, y_{q}\right) \mid t \geq \tau,\right. & \left|x_{1}\right|, \ldots,\left|x_{k}\right|,\left|y_{1}\right|, \ldots,\left|y_{l}\right| \leq M, \\
& \left|x_{k+1}\right|, \ldots,\left|x_{p}\right| \leq M e^{t}, \\
& \left.\left|y_{l+1}\right|, \ldots,\left|y_{q}\right| \leq M e^{2 t}\right\} .
\end{aligned}
$$

The volume of $\Lambda \cap N_{t}$ is comparable to $e^{-(2 k+l) t}$, as was to be shown.

\section{THE ERGODIC THEOREM}

For the following, observe that the unit tangent bundle $T$ inherits in a natural way a Riemannian metric from the base manifold $\mathcal{H}_{\mathbb{F}}^{n}$. Let $d_{T}$ denote the corresponding distance function on $T$ and define the distance function $d_{T / \Gamma}$ on $T / \Gamma$ by

$$
d_{T / \Gamma}\left(\Gamma v_{x}, \Gamma w_{y}\right)=\inf _{\gamma, \gamma^{\prime} \in \Gamma} d_{T}\left(\gamma v_{x}, \gamma^{\prime} w_{y}\right)
$$

Analogously, if $d$ is the hyperbolic distance on $\mathcal{H}_{\mathbb{F}}^{n}$, let us define a distance on $\mathcal{H}_{\mathbb{F}}^{n} / \Gamma$ by $d_{M_{\Gamma}}(\Gamma x, \Gamma y)=\inf _{\gamma, \gamma^{\prime} \in \Gamma} d\left(\gamma x, \gamma^{\prime} y\right)$.

Definition 5.1. We say that the geodesic flow on $T / \Gamma$ is conservative with respect to the invariant measure $\nu$ if almost every geodesic reenters a given bounded set in $T / \Gamma$ infinitely many times.

It is a well known result (see [As] for example), that if the geodesic flow $g_{t}$ on $T / \Gamma$ is conservative, then $\lim _{T \rightarrow \infty} \int_{0}^{T} \rho\left(g_{t} \cdot \Gamma v_{x}\right) d t=\infty$ for any positive function $\rho$ in $L^{1}(T / \Gamma, d \nu)$.

Theorem 5.2. If the geodesic flow on $T / \Gamma$ is conservative, then it is ergodic. 
Proof. The proof will follow the expositions of Hopf [Ho2] and Sullivan [Su1] and is recalled here for the sake of completeness.

If $f, \rho \in L^{1}(T / \Gamma, d \nu)$, let us define a function $f_{\rho}^{\tau}$ on $T / \Gamma$ by

$$
f_{\rho}^{\tau}\left(\Gamma v_{x}\right)=\frac{\int_{0}^{\tau} f\left(g_{t} \cdot \Gamma v_{x}\right) d t}{\int_{0}^{\tau} \rho\left(g_{t} \cdot \Gamma v_{x}\right) d t} .
$$

By the Birkhoff-Hopf ergodic theorem [Ho1], if the geodesic flow is conservative then the function $f_{\rho}^{\tau}$ satisfies the property that

(1) $\lim _{\tau \rightarrow+\infty} f_{\rho}^{\tau}\left(\Gamma v_{x}\right)=f_{\rho}^{+}\left(\Gamma v_{x}\right)$ and $\lim _{\tau \rightarrow-\infty} f_{\rho}^{\tau}\left(\Gamma v_{x}\right)=f_{\rho}^{-}\left(\Gamma v_{x}\right)$ exist for almost every $\Gamma v_{x}$ and are invariant for the action of the geodesic flow.

What we need to prove, to show ergodicity of $g_{t}$, is that $f_{\rho}^{+}=f_{\rho}^{-}$are constant almost everywhere, for every fixed positive $\rho \in L^{1}(T / \Gamma, d \nu)$ and every $f \in$ $L^{1}(T / \Gamma, d \nu)$. In order to prove this, we need some more technical consequences of the Birkhoff-Hopf ergodic theorem, precisely that

(2) $\rho f_{\rho}^{-}, \rho f_{\rho}^{+} \in L^{1}(T / \Gamma, d \nu)$ and moreover satisfy the property that for any bounded function $g$ on $T / \Gamma$ which is geodesic flow invariant

$$
\int_{T / \Gamma} \rho f_{\rho}^{+} d \nu=\int_{T / \Gamma} f g d \nu=\int_{T / \Gamma} \rho f_{\rho}^{-} d \nu
$$

Then a suitable choice of $g$ in (5.1) immediately implies that $f_{\rho}^{+}=f_{\rho}^{-}$almost everywhere.

To proceed with the proof, we shall use the following strategy. We can lift the function $f_{\rho}^{+}$to a function $\tilde{f}_{\rho}^{+}$on $T$, then, using the fibration by geodesics over $\left(\partial \mathcal{H}_{\mathbb{F}}^{n}\right)_{0}^{2}$, we push $\tilde{f}_{\rho}^{+}$down to a function on $\left(\partial \mathcal{H}_{\mathbb{F}}^{n}\right)_{0}^{2}$, after proving that the values of $\tilde{f}_{\rho}^{+}$depend only on the asymptotic value along each geodesic. Then a simple observation on sets which are unions of asymptotically equivalent geodesics (the measure theoretical technicalities of which we shall skip) will conclude the proof. Moreover, in the proof we shall be able to assume that $f$ is in a suitable dense subset of $L^{1}(T / \Gamma, d \nu)$, since an appropriate choice of $g$ in (5.1) shows that

$$
\int_{T / \Gamma} \rho\left|f_{\rho}^{+}\right| d \nu \leq\|f\|_{L^{1}(T / \Gamma, d \nu)}
$$

We shall hence work with the space $C_{c}(T / \Gamma, d \nu)$ of continuous functions on $T / \Gamma$ with compact support. Moreover, since for any positive $h \in L^{1}(T / \Gamma, d \nu)$ we have that $f_{h}^{\tau}=\frac{f_{\rho}^{\tau}}{h_{\rho}^{\tau}}$, it is clearly enough to prove that $f_{\rho}^{+}$is almost everywhere constant for a suitably chosen positive $\rho \in L^{1}(T / \Gamma, d \nu)$. In particular, we shall choose $\rho$ as in the following result, whose proof we postpone for a minute.

Lemma 5.3. The function $\rho$ on $T / \Gamma$ defined as

$$
\rho\left(\Gamma v_{x}\right)=e^{-C d_{M_{\Gamma}}(\Gamma x, \Gamma O)}
$$

where $O$ is the origin in $\mathcal{H}_{\mathbb{F}}^{n}$, is in $L^{1}(T / \Gamma, d \nu)$ for an appropriate choice of the constant $C$ and satisfies the inequality

$$
\frac{\rho\left(\Gamma w_{y}\right)}{\rho\left(\Gamma v_{x}\right)} \leq e^{k d_{T / \Gamma}\left(\Gamma v_{x}, \Gamma w_{y}\right)} .
$$

Continuation of the proof of Theorem 5.2. Assuming that $f \in C_{c}(T / \Gamma, d \nu)$ and $\rho \in L^{1}(T / \Gamma, d \nu)$ is as chosen above, we can now prove that $f_{\rho}^{+}$(and hence $\tilde{f}_{\rho}^{+}$) 
depends only on the asymptotic values of each geodesic; in other words, we can prove that if

$$
\lim _{t \rightarrow+\infty} g_{t} \cdot \Gamma v_{x}=\lim _{t \rightarrow+\infty} g_{t} \cdot \Gamma w_{y}
$$

then $f_{\rho}^{+}\left(\Gamma v_{x}\right)=f_{\rho}^{+}\left(\Gamma w_{y}\right)$.

Observe that because of (5.2), there exists a positive real number $h$ such that

$$
d_{T / \Gamma}\left(g_{t} \cdot \Gamma v_{x}, g_{t+h} \cdot \Gamma w_{y}\right) \rightarrow 0 \quad \text { as } t \rightarrow \infty .
$$

Moreover, from the fact that $\int_{0}^{\infty} \rho\left(g_{t} \cdot \Gamma v_{x}\right) d t=\infty$ it follows that

$$
\lim _{\tau \rightarrow \infty} \frac{\int_{0}^{\tau} f\left(g_{t} \cdot \Gamma w_{y}\right) d t}{\int_{0}^{\tau} \rho\left(g_{t} \cdot \Gamma w_{y}\right) d t}=\lim _{\tau \rightarrow \infty} \frac{\int_{0}^{\tau} f\left(g_{t+h} \cdot \Gamma w_{y}\right) d t}{\int_{0}^{\tau} \rho\left(g_{t+h} \cdot \Gamma w_{y}\right) d t} .
$$

Then we have

$$
\begin{aligned}
f_{\rho}^{+}\left(\Gamma v_{x}\right)-f_{\rho}^{+}\left(\Gamma w_{y}\right) & =\lim _{\tau \rightarrow \infty}\left(\frac{\int_{0}^{\tau} f\left(g_{t} \cdot \Gamma v_{x}\right) d t}{\int_{0}^{\tau} \rho\left(g_{t} \cdot \Gamma v_{x}\right) d t}-\frac{\int_{0}^{\tau} f\left(g_{t} \cdot \Gamma w_{y}\right) d t}{\int_{0}^{\tau} \rho\left(g_{t} \cdot \Gamma w_{y}\right) d t}\right) \\
& =\lim _{\tau \rightarrow \infty}\left(\frac{\int_{0}^{\tau} f\left(g_{t} \cdot \Gamma v_{x}\right) d t}{\int_{0}^{\tau} \rho\left(g_{t} \cdot \Gamma v_{x}\right) d t}-\frac{\int_{0}^{\tau} f\left(g_{t+h} \cdot \Gamma w_{y}\right) d t}{\int_{0}^{\tau} \rho\left(g_{t+h} \cdot \Gamma w_{y}\right) d t}\right) \\
& =\lim _{\tau \rightarrow \infty}\left(\frac{\int_{0}^{\tau}\left(f\left(g_{t} \cdot \Gamma v_{x}\right)-f\left(g_{t+h} \cdot \Gamma w_{y}\right)\right) d t}{\int_{0}^{\tau} \rho\left(g_{t} \cdot \Gamma v_{x}\right) d t}\right. \\
& \left.+\frac{\int_{0}^{\tau} f\left(g_{t+h} \cdot \Gamma w_{y}\right) d t}{\int_{0}^{\tau} \rho\left(g_{t+h} \cdot \Gamma w_{y}\right) d t} \cdot \frac{\int_{0}^{\tau} \frac{\rho\left(g_{t+h} \cdot \Gamma w_{y}\right)-\rho\left(g_{t} \cdot \Gamma v_{x}\right)}{\rho\left(g_{t} \cdot \Gamma v_{x}\right)} \rho\left(g_{t} \cdot \Gamma v_{x}\right) d t}{\int_{0}^{\tau} \rho\left(g_{t} \cdot \Gamma v_{x}\right) d t}\right) .
\end{aligned}
$$

When $t \rightarrow \infty$, if $d_{T / \Gamma}\left(g_{t} \cdot \Gamma v_{x}, g_{t+h} \cdot \Gamma w_{y}\right) \rightarrow 0$, then both

$$
f\left(g_{t} \cdot \Gamma v_{x}\right)-f\left(g_{t+h} \cdot \Gamma w_{y}\right) \rightarrow 0
$$

uniformly, since $f \in C_{c}(T / \Gamma, d \nu)$, and

$$
\frac{\rho\left(g_{t+h} \cdot \Gamma w_{y}\right)-\rho\left(g_{t} \cdot \Gamma v_{x}\right)}{\rho\left(g_{t} \cdot \Gamma v_{x}\right)} \leq e^{C d_{T / \Gamma}\left(g_{t} \cdot \Gamma v_{x}, g_{t+h} \cdot \Gamma w_{y}\right)}-1 \rightarrow 0
$$

uniformly, because of the choice of $\rho$ as in Lemma 5.3. Hence, it follows from the hypothesis on $g_{t}$ and (5.3) that both

$$
\lim _{\tau \rightarrow \infty} \frac{\int_{0}^{\tau}\left(f\left(g_{t} \cdot \Gamma v_{x}\right)-f\left(g_{t+h} \cdot \Gamma w_{y}\right)\right) d t}{\int_{0}^{\tau} \rho\left(g_{t} \cdot \Gamma v_{x}\right) d t}=0
$$

and

$$
\lim _{\tau \rightarrow \infty} \frac{\int_{0}^{\tau} \frac{\rho\left(g_{t+h} \cdot \Gamma w_{y}\right)-\rho\left(g_{t} \cdot \Gamma v_{x}\right)}{\rho\left(g_{t} \cdot \Gamma v_{x}\right)} \rho\left(g_{t} \cdot \Gamma v_{x}\right) d t}{\int_{0}^{\tau} \rho\left(g_{t} \cdot \Gamma v_{x}\right) d t}=0 .
$$

To conclude this part of the proof all we need to do is to observe that

$$
\frac{\int_{0}^{\tau} f\left(g_{t+h} \cdot \Gamma w_{y}\right) d t}{\int_{0}^{\tau} \rho\left(g_{t+h} \cdot \Gamma w_{y}\right) d t}
$$

is bounded as $\tau \rightarrow \infty$. In fact, $\rho$ is bounded below on compact sets so that $f / \rho \leq C_{1}$, and hence

$$
\frac{\int_{0}^{\tau} f\left(g_{t+h} \cdot \Gamma w_{y}\right) d t}{\int_{0}^{\tau} \rho\left(g_{t+h} \cdot \Gamma w_{y}\right) d t} \leq C_{1}
$$




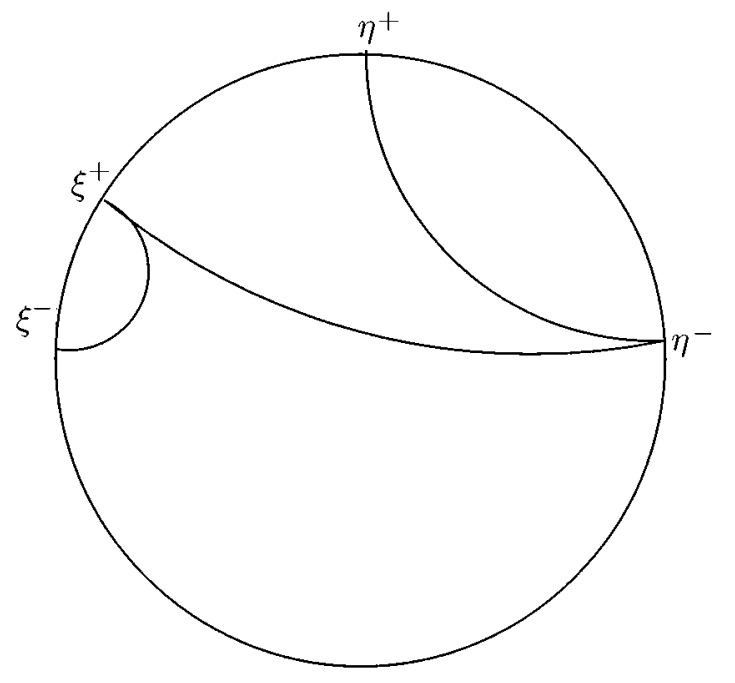

FigURE 3

To conclude the proof, observe that if $B^{+}$and $B^{-}$are geodesic flow invariant subsets of $T$ which are unions of equivalence classes of (respectively) positively and negatively asymptotic geodesics, with the additional property that the measure of their symmetric difference is zero, then either both $B^{+}$and $B^{-}$are null or they are both conull. We shall omit the proof of this statement (which can be found in [Ho2]) and illustrate instead why the above assertion is true if we replace the property of having measure zero with the property of being the empty set. In fact, in this case, we have that $B^{+}=B^{-}$and the statement is that $B^{+}=B^{-}$is either empty or the whole $T$. If it is not empty, let $\sigma \approx\left(\xi^{-}, \xi^{+}\right) \in B^{+}$denote the geodesic such that $\lim _{t \rightarrow \pm \infty} \sigma(t)=\xi^{ \pm} \in \partial \mathcal{H}_{\mathbb{F}}^{n}$. If $\sigma_{1} \approx\left(\eta^{-}, \eta^{+}\right)$is any other geodesic in $T$, then there exists a unique geodesic $\sigma_{2} \approx\left(\eta^{-}, \xi^{+}\right)$, hence implying that $\sigma, \sigma_{1}, \sigma_{2} \in B^{+}$. See Figure 3.

In our case, let us define

$$
B^{+}=\left\{\Gamma v_{x}: \tilde{f}_{\rho}^{+}\left(\Gamma v_{x}\right) \geq C_{0}\right\} \quad \text { and } \quad B^{-}=\left\{\Gamma v_{x}: \tilde{f}_{\rho}^{-}\left(\Gamma v_{x}\right) \geq C_{0}\right\} .
$$

Then, by what was proven above, both $B^{+}$and $B^{-}$are either null or conull and, being true for any constant $C_{0}$, this implies that $f_{\rho}^{+}=f_{\rho}^{-}$are constant almost everywhere, hence concluding the proof.

Proof of Lemma 5.3. Since the differential of $d_{M_{\Gamma}}$ has absolute value at most 1, $\left|\frac{d \rho}{\rho}\right| \leq C$ and hence integration along any path $\pi$ connecting $\Gamma v_{x}$ and $\Gamma w_{y}$ gives

$$
C l(\pi) \geq \int_{\pi} \frac{d \rho}{\rho}=\int_{\pi} d \log \rho=\log \rho\left(\Gamma w_{y}\right)-\log \rho\left(\Gamma v_{x}\right) .
$$

Since this inequality holds for any such path, we have

$$
\log \frac{\rho\left(\Gamma w_{y}\right)}{\rho\left(\Gamma v_{x}\right)} \leq C d_{T / \Gamma}\left(\Gamma v_{x}, \Gamma w_{y}\right)
$$

from which the inequality of the statement follows. 


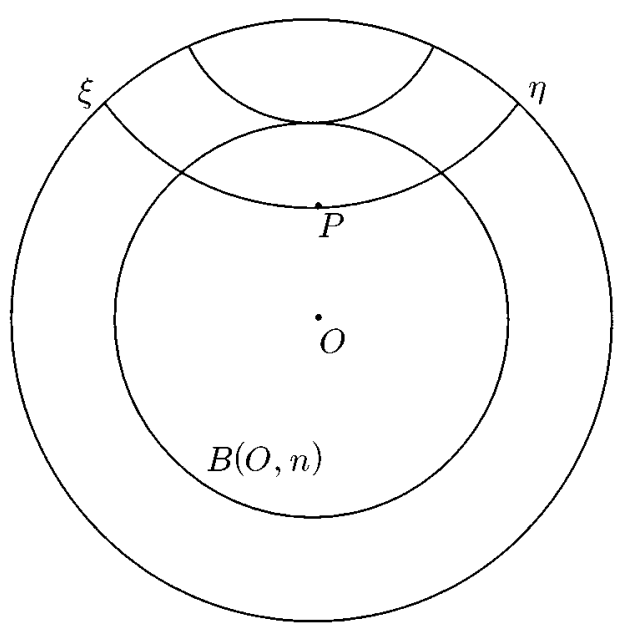

Figure 4

We need to check now that $\rho \in L^{1}(T / \Gamma, d \nu)$ for an appropriate choice of the constant $C$. In order to do this, we shall estimate the $L^{1}$-norm of $\rho$ by means of an infinite discrete sum. Let us define $\Sigma_{n} \subset T / \Gamma$ to be $\Sigma_{n}=\Delta^{-1}[0, n]$, where $\Delta: T / \Gamma \rightarrow \mathbb{R}^{+}$is defined in terms of the distance function on $M_{\Gamma}$ by $\Delta\left(\Gamma v_{x}\right)=$ $d_{M_{\Gamma}}(\Gamma x, \Gamma O)$; in other words, $\Delta$ measures the distance of the base point of a tangent vector from the orbit of the origin in $\mathcal{H}_{\mathbb{F}}^{n}$. Then we clearly have

$$
\|\rho\|_{L^{1}} \leq \sum_{n=1}^{\infty} \rho_{n} \nu\left(\Sigma_{n} \backslash \Sigma_{n-1}\right) \leq \sum_{n=1}^{\infty} \rho_{n} \nu\left(\Sigma_{n}\right)
$$

where $\rho_{n}=\max \left\{\rho\left(\Gamma v_{x}\right): \Gamma v_{x} \in \Sigma_{n} \backslash \Sigma_{n-1}\right\}=e^{-C(n-1)}$. We need to give now an explicit estimate of $\nu\left(\Sigma_{n}\right)$ using the definition of $\nu$ and hence of $\tilde{\nu}$ given at the beginning of $\S 4$. Aiming for this, we offer a few simple observations: if $q: T \rightarrow T / \Gamma$ is the natural projection, by definition of $\nu$ we have that $\nu\left(\Sigma_{n}\right)=\tilde{\nu}\left(\tilde{\Sigma}_{n}\right)$, where $\tilde{\Sigma}_{n} \subset T$ is any set such that $q\left(\tilde{\Sigma}_{n}\right)=\Sigma_{n}$. For our purpose however, since we are interested just in an upper bound for $\nu\left(\Sigma_{n}\right)$, it will be convenient to choose $\tilde{\Sigma}_{n}=\left\{v_{x} \in T: x \in \overline{B(O, n)}\right\}$, where $\overline{B(O, n)}$ is the closure of $B(O, n)=\left\{x \in \mathcal{H}_{\mathbb{F}}^{n}\right.$ : $d(O, x)<n\}$. Then clearly $q\left(\tilde{\Sigma}_{n}\right) \supset \Sigma_{n}$ and $\nu\left(\Sigma_{n}\right) \leq \tilde{\nu}\left(\tilde{\Sigma}_{n}\right)$. Moreover, recalling the definition of $\tilde{\nu}$ and observing that the length of an arc of a geodesic through $B(O, n)$ is at most $2 n$, we have that $\tilde{\nu}\left(\tilde{\Sigma}_{n}\right) \leq 2 n \cdot m\left(p\left(\tilde{\Sigma}_{n}\right)\right)$, where $p: T \rightarrow\left(\partial \mathcal{H}_{\mathbb{F}}^{n}\right)_{0}^{2}$ is the fibration map.

To estimate $m\left(p\left(\tilde{\Sigma}_{n}\right)\right)$ observe that if $(\xi, \eta) \in\left(\partial \mathcal{H}_{\mathbb{F}}^{n}\right)_{0}^{2}$ and $\sigma$ is a geodesic in $\mathcal{H}_{\mathbb{F}}^{n}$ such that $\lim _{t \rightarrow \infty} \sigma(-t)=\xi$ and $\lim _{t \rightarrow \infty} \sigma(t)=\eta$, then

$$
-f_{O}(\xi, \eta)=\lim _{t \rightarrow \infty}(d(O, \sigma(t))+d(\sigma(-t), O)-d(\sigma(-t), \sigma(t))) \leq 2 d(O, P)
$$

where $P \in \mathcal{H}_{\mathbb{F}}^{n}$ is the point which minimizes the distance of $\sigma$ from the origin $O \in \mathcal{H}_{\mathbb{F}}^{n}$. See Figure 4 .

Since we are interested only in the geodesics through $\tilde{\Sigma}_{n}$, we get that $0 \leq$ $-f_{O}(\xi, \eta) \leq 2 n$ as $(\xi, \eta) \in p\left(\tilde{\Sigma}_{n}\right)$ (where $2 n$ is the value of $-f_{O}(\xi, \eta)$ corresponding to the limit position, that is when $\sigma$ is tangent to $\overline{B(O, n)})$. Hence 
$m\left(p\left(\tilde{\Sigma}_{n}\right)\right)=m_{O}\left(p\left(\tilde{\Sigma}_{n}\right)\right) \leq \mu_{O}\left(L_{\Gamma}\right)^{2} e^{2 n \delta_{\Gamma}}$. We can thus conclude that

$$
\begin{aligned}
\|\rho\|_{L^{1}(T / \Gamma, d \nu)} & \leq \sum_{n=1}^{\infty} \rho_{n} \nu\left(\Sigma_{n}\right) \leq \sum_{n=1}^{\infty} \rho_{n} \tilde{\nu}\left(\tilde{\Sigma}_{n}\right) \\
& \leq \sum_{n=1}^{\infty} e^{-C(n-1)} \cdot 2 n \cdot \mu_{O}\left(L_{\Gamma}\right)^{2} \cdot e^{2 n \delta_{\Gamma}}=2 C^{\prime} \sum_{n=1}^{\infty} n e^{2 \delta_{\Gamma} n-C n},
\end{aligned}
$$

which converges if $C=2 \delta_{\Gamma}+\epsilon$, with $\epsilon>0$.

Proposition 5.4. The geodesic flow is conservative.

As an immediate consequence of Theorem 5.2 and Proposition 5.4 we have the following:

Corollary 5.5. The geodesic flow is ergodic.

Proof of Proposition 5.4. It follows from Theorem 3.2 that the set of geodesics with both endpoints in $L_{\Gamma}^{\mathrm{rad}}$ is a set of full measure. But for these geodesics the existence of a bounded neighborhood with the desired property follows immediately from the definition of radial limit point.

Before ending this section we want to prove a result which will be used in the proof of Theorem 6.1.

Corollary 5.6 [Su1]. For any $v_{x} \in T$, let $\sigma$ be the geodesic such that $\sigma(0)=x$ and $\sigma^{\prime}(0)=v_{x}$. Then, for almost every $v_{x} \in T$,

$$
\lim _{t \rightarrow \infty} \frac{1}{t} d_{M_{\Gamma}}(\Gamma \sigma(t), \Gamma 0)=0
$$

Proof. Let $\varphi(v)$ be the directional derivative, in the direction of $v$, of the function $\Delta\left(\Gamma v_{x}\right)=d_{M_{\Gamma}}(\Gamma x, \Gamma 0)$ defined in the proof of Lemma 5.3. Since $\mu_{x}$ has no atoms, the geodesic flow on $T / \Gamma$ is conservative and therefore ergodic. Moreover, as shown in Theorem 4.1, $\nu(T / \Gamma)<\infty$. We can thus apply the Birkhoff ergodic theorem which implies that

$$
\lim _{t \rightarrow \infty} \frac{1}{t}\left(d_{M_{\Gamma}}(\Gamma \sigma(t), \Gamma 0)-d_{M_{\Gamma}}(\Gamma x, \Gamma 0)\right)=\lim _{t \rightarrow \infty} \frac{1}{t} \int_{0}^{t} \varphi\left(g_{s} v_{x}\right) d s=\int_{T / \Gamma} \varphi d \nu .
$$

It will be hence enough to show that $\int_{T / \Gamma} \varphi d \nu=0$. To this end, let us observe that the map $I: T \rightarrow T$ defined by $I\left(v_{x}\right)=-v_{x}$ preserves the measure $m$ on $\left(\partial \mathcal{H}_{\mathbb{F}}^{n}\right)_{0}^{2}$ and commutes with the action of $\Gamma$, so that it preserves the measure $\nu$. Moreover, clearly $\varphi \circ I=-\varphi$, so that

$$
\int_{T / \Gamma} \varphi d \nu=\int_{T / \Gamma}(\varphi \circ I) d \nu(I)=-\int_{T / \Gamma} \varphi d \nu(I)=-\int_{T / \Gamma} \varphi d \nu
$$

which is what we wanted.

Remark. The hypothesis on the absence of atoms for $\mu_{x}$ in Corollary 5.6 could be replaced by the $\delta_{\Gamma}$-finiteness of the volume. In fact it can be easily shown using the Poincaré Recurrence Theorem that if $\nu(T / \Gamma)<\infty$ the geodesic flow is conservative and hence ergodic. 


\section{HAUSDORFF DIMENSION AND CRITICAL EXPONENT}

Theorem 6.1. Let $\Gamma$ be a geometrically finite discrete subgroup of $O_{\mathbb{F}}(1, n)$. Then the Hausdorff dimension of the radial limit set $L_{\Gamma}^{r a d}$, and hence of the full limit set $L_{\Gamma}$, is equal to the critical exponent $\delta_{\Gamma}$.

Before proving this, we want to note here a few results which are a direct consequence of this theorem. The first corollary was already proven by Sullivan in the particular case of the real hyperbolic space [Su3].

Corollary 6.2. Let $\Delta_{M_{\Gamma}}$ be the Laplace operator on $C^{\infty}\left(M_{\Gamma}\right) \cap L^{2}\left(M_{\Gamma}\right)$ and let $\lambda_{0}$ be the infimum of the spectrum of $\Delta_{M_{\Gamma}}$. Then $\lambda_{0}=\delta_{\Gamma}\left(N-\delta_{\Gamma}\right)$, where $N$ is the Hausdorff dimension of $\partial \mathcal{H}_{\mathbb{F}}^{n}$.

Proof. Theorem 6.1 and [C, Theorem 4.2].

Corollary 6.3. Let $G$ be $O_{\mathbb{H}}(1, n), n \geq 2$ or $O_{\mathbb{O}}(1,2)$ and let $\alpha=4 n$ or $\alpha=16$ respectively. If $\Gamma \subset G$ is geometrically finite but is not a lattice, the Hausdorff dimension of the limit set is at most $\alpha$.

Proof. Corollary 2.4, Theorem 6.1 and [C, Theorem 4.4].

Corollary 6.4. Let $G$ be $O_{\mathbb{H}}(1, n), n \geq 2$, or $O_{\mathbb{O}}(1,2)$ and let $\Gamma \subset G$ be geometrically finite. Then $O_{\Gamma}$ is connected. In particular $M_{\Gamma}$ has at most one end which is not a cusp, i.e. $M_{\Gamma}$ may have several ends at most one of which cannot be identified with the quotient of a horoball by a discrete parabolic group.

Proof. Corollary 2.4 and the proof of [C, Theorem 7.1].

Proof of Theorem 6.1. In [C, Theorem 5.2] it is already proven that $\delta_{\Gamma}$ is an upper bound for the Hausdorff dimension of $L_{\Gamma}^{\text {rad }}$. We are going to show here the reverse inequality. We claim that it will be enough to prove the following:

Lemma 6.5. Let $\mu_{x}$ be the Patterson-Sullivan measure, $K \subset L_{\Gamma}^{\text {rad }}$ a compact set of positive $\mu_{x}$-measure and $B_{\partial \mathcal{H}_{\mathrm{F}}^{n}}(\xi, r)$ a ball of radius $r$ and center $\xi \in K$ in a sub-Riemannian metric in the given conformal class. Then for any $\epsilon>0$ there exists a constant $C(\epsilon)$ such that

$$
\frac{\mu_{x}\left(B_{\partial \mathcal{H}_{\mathbb{F}}^{n}}(\xi, r)\right)}{r^{\delta_{\Gamma}-\epsilon}} \leq C(\epsilon) .
$$

We postpone the proof of this lemma for a minute, and we show how it implies Theorem 6.1. We want to show that for any $\epsilon>0$, the $\left(\delta_{\Gamma}-\epsilon\right)$-Hausdorff measure of $L_{\Gamma}^{\mathrm{rad}}$ is positive or, in fact, that the $\left(\delta_{\Gamma}-\epsilon\right)$-Hausdorff measure of $K$ is positive. To this end, let us consider a covering $\left\{B_{\partial \mathcal{H}_{\mathbb{F}}^{n}}^{i}\left(\xi_{i}, r_{i}\right)\right\}$ of $K$, where $\xi_{i} \in K$. Then, by Lemma 6.5 ,

$$
0<\mu_{x}(K) \leq \mu_{x}\left(\bigcup_{i} B_{\partial \mathcal{H}_{\mathbb{P}}^{n}}^{i}\left(\xi_{i}, r_{i}\right)\right) \leq \sum_{i} \mu_{x}\left(B_{\partial \mathcal{H}_{\mathbb{P}}^{n}}^{i}\left(\xi_{i}, r_{i}\right)\right) \leq C \sum_{i} r_{i}^{\delta_{\Gamma}-\epsilon},
$$

which is exactly what we wanted.

Proof of Lemma 6.5. The strategy will be to estimate the measure of the ball $B_{\partial \mathcal{H}_{F}^{n}}(\xi, r)$ via the measure of some larger shadow.

Fix $\epsilon>0$ and let $\sigma$ be the geodesic ray from $x \in \mathcal{H}_{\mathbb{F}}^{n}$ towards $\xi \in \partial \mathcal{H}_{\mathbb{F}}^{n}$. Because of Theorem 3.6 and Corollary 5.6 there exists $T>0$ such that if $t>T$, then

$$
\delta_{\Gamma} \inf _{\gamma \in \Gamma} d(\sigma(t), \gamma y)<\epsilon t
$$




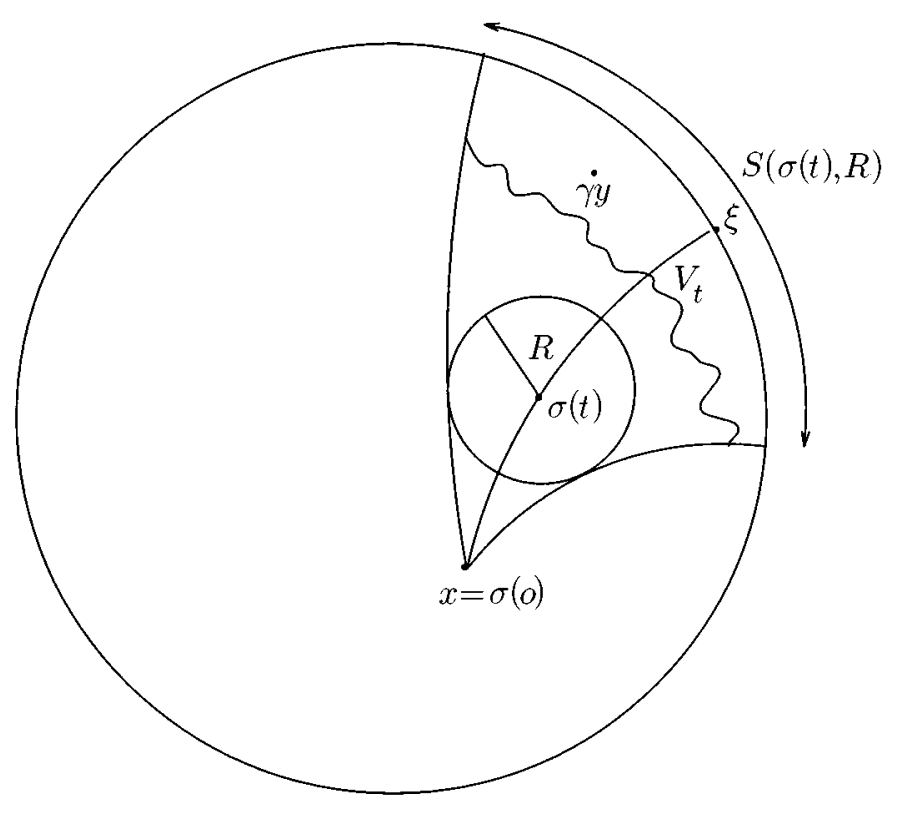

FiguRE 5

where $y \in \mathcal{H}_{\mathbb{F}}^{n}$ is some arbitrarily fixed point. Fix some $t$ which satisfies the above inequality. Given a ball $B(\sigma(t), R) \subset \mathcal{H}_{\mathbb{F}}^{n}$ of hyperbolic radius $R<t$ and centered at $\sigma(t)$, the shadow of such a ball is defined as the subset $S(\sigma(t), R)$ of $\partial \mathcal{H}_{\mathbb{F}}^{n}$ consisting of endpoints of geodesic rays starting at $x$ through $B(\sigma(t), R)$ and has the property that there exist constants $C_{1}$ and $C_{2}$ such that $B_{\partial \mathcal{H}_{\mathbb{R}}^{n}}\left(\xi, C_{1} e^{-t}\right) \subset S(\sigma(t), R) \subset$ $B_{\partial \mathcal{H}_{\mathrm{F}}^{n}}\left(\xi, C_{2} e^{-t}\right)$, by [C, Theorem 2.2]. Now choose the radius $R$ in such a way that $C_{1} e^{-t}=r$. It will hence suffice to show that

$$
\frac{\mu_{x}(S(\sigma(t), R))}{r^{\delta_{\Gamma}-\epsilon}}<C(\epsilon) .
$$

Let $V_{t}$ be the region in the cone of geodesics starting at $x$ through $B(\sigma(t), R)$ characterized by the property that $\inf _{z \in V_{t}} d(x, z)>t$. From Proposition 3.5 and the definition of Patterson-Sullivan measure, it follows that

$$
\mu_{x}(S(\sigma(t), R))=\lim _{s \rightarrow \delta_{\Gamma}} \frac{\sum_{\gamma y \in V_{t}} e^{-s d(x, \gamma y)}}{\sum_{\gamma \in \Gamma} e^{-s d(y, \gamma y)}},
$$

and we want to estimate $\sum_{\gamma y \in V_{t}} e^{-s d(x, \gamma y)}$. See Figure 5 .

To this end, observe that if $\gamma y \in V_{t}$, then the angle between $\sigma$ and the arc of a geodesic connecting $\sigma(t)$ to $\gamma y$ is bounded away from 0 , so that we can apply Lemma 3.1 to the triangle with vertices at the points $x, \sigma(t), \gamma y$ to see that

$$
d(x, \sigma(t))+d(\sigma(t), \gamma y)-C_{3} \leq d(x, \gamma y) \leq d(x, \sigma(t))+d(\sigma(t), \gamma y) .
$$

This, together with the triangle inequality, gives us

$$
-d(x, \gamma y) \leq-t-d(\sigma(t), \gamma y)+C_{3} \leq-t-d\left(\gamma y, \gamma_{0} y\right)+d\left(\gamma_{0} y, \sigma(t)\right)+C_{3},
$$


where $\gamma_{0} y$ is the point (not necessarily unique) which minimizes the distance of $\sigma(t)$ from $\Gamma y$. Hence

$$
\begin{aligned}
\mu_{x}(S(\sigma(t), R))= & \lim _{s \rightarrow \delta_{\Gamma}} \frac{\sum_{\gamma y \in V_{t}} e^{-s d(x, \gamma y)}}{\sum_{\gamma \in \Gamma} e^{-s d(y, \gamma y)}} \\
& \leq \lim _{s \rightarrow \delta_{\Gamma}} \frac{e^{-s t+s C_{3}+s d\left(\sigma(t), \gamma_{0} y\right)} \sum_{\gamma y \in V_{t}} e^{-s d\left(\gamma y, \gamma_{0} y\right)}}{\sum_{\gamma \in \Gamma} e^{-s d(y, \gamma y)}} \\
& \leq e^{-\delta_{\Gamma} t+\delta_{\Gamma} C_{3}+\delta_{\Gamma} d\left(\sigma(t), \gamma_{0} y\right)} \lim _{s \rightarrow \delta_{\Gamma}} \frac{\sum_{\gamma \in \Gamma} e^{-s d\left(\gamma y, \gamma_{0} y\right)}}{\sum_{\gamma \in \Gamma} e^{-s d(y, \gamma y)}} \\
& =e^{-\delta_{\Gamma} t+\delta_{\Gamma} C_{3}+\delta_{\Gamma} d\left(\sigma(t), \gamma_{0} y\right)}=C_{4} e^{-\delta_{\Gamma} t+\delta_{\Gamma} d\left(\sigma(t), \gamma_{0} y\right)}
\end{aligned}
$$

Because of our choice of $t$ and recalling that $C_{1} e^{-t}=r$ (where $C_{1}$ depends on $\epsilon$ and $\xi$ ), we can hence conclude that

$$
\begin{aligned}
\mu_{x}(S(\sigma(t), R)) & \leq C_{4} e^{-\delta_{\Gamma} t+\delta_{\Gamma} d\left(\sigma(t), \gamma_{0} y\right)} \leq C_{4} e^{-\left(\delta_{\Gamma}-\epsilon\right) t} \\
& =\frac{C_{4}}{C_{1}(\epsilon, \xi)^{\delta_{\Gamma}-\epsilon}} r^{\delta_{\Gamma}-\epsilon} \leq C_{5}(\epsilon) r^{\delta_{\Gamma}-\epsilon},
\end{aligned}
$$

where the last step is possible because $\xi$ varies in a compact set.

\section{REFERENCES}

[Ad] Anderson, M., The Dirichlet problem at infinity, J. Diff. Geom. 18 (1983), 701-721. MR 85m:58178

[As] Anosov, D. V., Geodesic flows on closed Riemannian manifolds with negative curvature, Proceedings of the Steklov Institute of Mathematics, vol. 90, A. M. S., Providence, RI, 1969. MR 39:3527

[Au] Auslander, L., Bieberbach's theorem on space groups and discrete uniform subgroups of Lie groups, II, Amer. J. Math. 83 (1961), 276-280. MR 23:A292

[Bi-J] Bishop, C. and Jones, P., Hausdorff dimension and Kleinian groups, preprint.

[Bu] Bourdon, M., Structure conforme au bord et flot géodésique d' un CAT(-1)-espace, L' Enseignement Math. 41 (1995), 63-102. MR 96f:58120

[Bw] Bowditch, B. H., Geometrical finiteness with variable curvature, J. Func. An. 113 (1993), 245-317. MR 94e:57016

[C] Corlette, K., Hausdorff dimensions of limit sets I, Invent. Math. 102 (1990), 521-542. MR 91k:58067

[E-O] Eberlein, P. and O'Neill, B., Visibility manifolds, Pac. J. Math. 46 (1973), 45-109. MR 49:1421

[Go] Goldman, W., A user's guide to complex hyperbolic geometry, Oxford Math. Monographs (to appear).

[Gr] Gromov, M., Asymptotic geometry of homogeneous spaces, Conference on Differential geometry on homogeneous spaces (Torino, 1983), Rend. Sem. Mat. Univ. Politec. Torino 1983, Fasc. Spec. 59-60 (1984). CMP 18:09

[He1] Helgason, S., Groups and geometric analysis, Academic Press, New York, 1984. MR 86c: 22017

[He2] — Differential geometry, Lie groups and symmetric spaces, Academic Press, New York, 1989. MR 80k:53081

[Ho1] Hopf, E., Ergodentheorie, Ergebnisse der Mathematik, Band 5, no.2, Springer-Verlag, 1937.

[Ho2] _ Ergodic theory and the geodesic flow on surfaces of constant negative curvature, Bull. Am. Math. Soc. 77 (1971), 863-877. MR 44:1789

[K] Kaimanovich, V. A., Invariant measures for the geodesic flow and measures at infinity on negatively curved manifolds, Ann. Inst. Henri Poincaré, Physique Théorique 53 (4) (1990), 361-393. MR 92b:58176

[Mi] Mitchell, J., On Carnot-Carathéodory metrics, J. Diff. Geom. 21 (1985), 35-45. MR 87d:53086 
[Mo] Mostow, G., Strong rigidity of locally symmetric spaces, vol. 78, Ann. Math. Stud., Princeton University Press, Princeton, 1978. MR 52:5874

[Pn1] Pansu, P., Thèse.

[Pn2] _ Une inégalité isopérimétrique sur le groups de Heisenberg, C. R. Acad. Sci. Paris Sér. I295 (1982), 127-130. MR 85b:53044

[Pn3] - Metrique de Carnot-Carathéodory et quasi-isométries des espaces symmetrique de rang un, Ann. Math. 129 (1989), 1-60. MR 90e:53058

[Pt] Patterson, S. J., The limit set of a Fuchsian group, Acta Math. 136 (1976), 241-273. MR 56:8841

[St] Strichartz, R., Sub-Riemannian geometry, J. Diff. Geom. 24 (1980), 221-263. MR 88b:53055

[Su1] Sullivan, D., The density at infinity of a discrete group of hyperbolic motions, Publ. I.H.E.S. 50 (1979), 171-202. MR 81b:58031

[Su2] Entropy, Hausdorff measures old and new, and limit sets of geometrically finite Kleinian groups, Acta Math. 153 (1984), 259-277. MR 86c:58093

[Su3] _ Related aspects of positivity in Riemannian manifolds, J. Diff. Geom. 25 (1987), 327-351. MR 88d:58132

[Y] Yue, C., The ergodic theory of discrete isometry groups of manifolds of variable negative curvature, preprint.

[W] Wang, H., Discrete subgroups of solvable Lie groups I, Ann. of Math. 64(1) (1956), 1-19. MR 17:1224c

Department of Mathematics, University of Chicago, Chicago, Illinois 60637

E-mail address: kevin@math.uchicago.edu

Department of Mathematics, University of Maryland, College Park, Maryland 20742

E-mail address: iozzi@math.umd.edu 